\title{
Integrable Quantum Systems and Classical Lie Algebras
}

\author{
V. V. Bazhanov \\ Institute for High Energy Physics, Serpukhov, Moscow Region, USSR
}

\begin{abstract}
We have obtained six new infinite series of trigonometric solutions to triangle equations (quantum $R$-matrices) associated with the nonexceptional simple Lie algebras: $s l(N), s p(N), o(N)$. The $R$-matrices are given in two equivalent representations: in an additive one (as a sum of poles with matrix coefficients) and in a multiplicative one (as a ratio of entire matrix functions). These $R$-matrices provide an exact integrability of anisotropic generalizations of $\operatorname{sl}(N), \operatorname{sp}(N), o(N)$ invariant one-dimensional lattice magnetics and twodimensional periodic Toda lattices associated with the above algebras.
\end{abstract}

\section{Table of Contents}

1. Introduction . . . . . . . . . . . . . . . . . . . 471

2. Some Information from the Theory of Simple Lie Algebras . . . . . . . . . . . 475

3. Classical Triangle Equations . . . . . . . . . . . . . . . . . . . . . . . . 477

3.1. Classical $r$-Matrices . . . . . . . . . . . . . . . . . . . . 477

3.2. Classical Toda Lattices. . . . . . . . . . . . . . . . . . . . . . . . . . 480

4. Quantum Triangle Equations . . . . . . . . . . . . . . . . . . . . . 481

4.1. A Simple Theorem . . . . . . . . . . . . . . . . . . . . . 481

4.2. Quantization of Trigonometric $r$-Matrices . . . . . . . . . . . . . . . . . 482

5. Integrable Quantum Systems . . . . . . . . . . . . . . . . . . . . . . . . 484

5.1. Quantum Toda Lattices . . . . . . . . . . . . . . . . . . . . . . . . 484

5.2. Integrable Models of Magnetics . . . . . . . . . . . . . . . . . . . . . 487

6. Factorized Representations for Quantum R-Matrices . . . . . . . . . . . . . . 487

6.1. Preliminary Remarks . . . . . . . . . . . . . . . . . . . . . . . . . . 487

6.2. Matrix Generalizations of Trigonometric Functions . . . . . . . . . . . . 487

6.3. Two Types of $\zeta$ - and $\sigma$-Functions . . . . . . . . . . . . . . . . . . . . . . . . . . . 489

6.4. Factorization of Quantum $R$-Matrices . . . . . . . . . . . . . . . . . . . 490

Appendix A. . . . . . . . . . . . . . . . . . . . . . . . . . . 493

Appendix B. . . . . . . . . . . . . . . . . . . . . . . . . . . . 494

Appendix C . . . . . . . . . . . . . . . . . . . . . . . . . 495

Appendix D . . . . . . . . . . . . . . . . . . . . . . . . . . . . . 497

References . . . . . . . . . . . . . . . . . . . . 501

\section{Introduction}

In the theory of two-dimensional integrable systems of quantum field theory and statistical physics a specific importance is attached to the special system of 
algebraic functional equations called triangle equations (or Yang-Baxter equations)

$$
R_{i_{1} i_{2}}^{j_{1} j_{2}}(\theta) R_{j_{1} i_{3}}^{k_{1} j_{3}}\left(\theta+\theta^{\prime}\right) R_{j_{2} j_{3}}^{k_{2} k_{3}}\left(\theta^{\prime}\right)=R_{i_{2} i_{3}}^{j_{2} j_{3}}\left(\theta^{\prime}\right) R_{i_{1} j_{3}}^{j_{1} k_{3}}\left(\theta+\theta^{\prime}\right) R_{j_{1} j_{2}}^{k_{1} k_{2}}(\theta) .
$$

Here $\theta, \theta^{\prime}$ are complex variables, the indices run over $N$ various values. The summation over the repeated indices is assumed.

Each solution of the triangle Eqs. (1.1) can be treated, on the one hand, as an exact factorized $S$-matrix in some $1+1$-dimensional field theory $[1,2]$ and, on the other hand, as a vertex weight matrix of an exactly soluble statistical model on plane lattice $[3,4]$. Besides, the triangle equation arises as the consistency condition for the Bethe anzatz solution of quantum-field models [5-7] and those of one-dimensional magnetics [7]. Triangle equations also make a part of the quantum inverse problem technique $[8,9]$. Finally, studies of triangle equations resulted in a new mathematical object called quantum groups [10].

Thus, each solution of the triangle equations is associated with quite a number of exactly soluble models from field theory and lattice statistics.

Usually Eq. (1.1) is written in a more compact form using matrix notations. We shall consider $R_{i_{1} i_{2}}^{j_{1} j_{2}}(\theta)$ as matrix elements of some matrix $R(\theta)$ acting in the tensor product of two vector spaces $\mathbb{C}^{N} \otimes \mathbb{C}^{N}$. The matrix $R(\theta)$ is called a (quantum) $R$-matrix. Introduce the matrices $R_{12}(\theta), R_{13}(\theta), R_{23}(\theta)$, acting in the tensor product of three vector spaces $\mathbb{C}^{N} \otimes \mathbb{C}^{N} \otimes \mathbb{C}^{N}$ according to the rule

$$
\left(R_{12}\right)_{i_{1} i_{2} i_{3}}^{j_{1} j_{2} j_{3}}=R_{i_{1} i_{2}}^{j_{1} j_{2}}(\theta) \delta_{i_{3}}^{i_{3}}
$$

$\left[R_{13}(\theta)\right.$ and $R_{23}(\theta)$ are defined similarly, they act identically in the second and first spaces, respectively]. In new notations, Eqs. (1.1) become

$$
R_{12}(\theta) R_{13}\left(\theta+\theta^{\prime}\right) R_{23}\left(\theta^{\prime}\right)=R_{23}\left(\theta^{\prime}\right) R_{13}\left(\theta+\theta^{\prime}\right) R_{12}(\theta) .
$$

The quantum $R$-matrix is called quasi-classical if it depends on the additional parameter $\varphi$ (playing the role of the Planck constant) so that for small $\varphi$,

$$
R(\theta, \varphi)=1+2 \varphi r(\theta)+O\left(\varphi^{2}\right) .
$$

Substituting (1.2) into $\left(1.1^{\prime}\right)$, one obtains the classical triangle equations, quite essential for the theory of integrable classical systems [11]:

$$
\left[r_{12}(\theta), r_{13}\left(\theta+\theta^{\prime}\right)+r_{23}\left(\theta^{\prime}\right)\right]+\left[r_{13}\left(\theta+\theta^{\prime}\right), r_{23}\left(\theta^{\prime}\right)\right]=0
$$

$[$,$] denoting a commutator. The quantity r(\theta)$ is called a classical $r$-matrix.

Note, that Eq. (3) is written only with the help of commutators. Therefore, one may assume that $r \in \mathscr{G} \otimes \mathscr{G}, \mathscr{G}$ being a Lie algebra. It becomes clear then that the solutions of (3) can be written in an invariant form, i.e. independent of the representation of $\mathscr{G}$. However, the corresponding solutions to the quantum Eqs. (1.1) depend essentially on the representation of $\mathscr{G}$.

Intensive studies [12-24] of Eqs. (1.1) and (1.3) over the last years led to the discovery of a great number of new integrable models and provided extensive "experimental" material that has clarified essentially the general structure of $R$-matrices. Let us enumerate here some characteristic properties of $R$-matrices.

(i) All known $R$-matrices are meromorphic functions of $\theta$ expressed via rational, trigonometric, or elliptic functions only. 
(ii) Trigonometric and elliptic $R$-matrices possess an automorphicity property. In the trigonometric case, this property is described as

$$
R(\theta+\pi)=(U \otimes 1) R(\theta)(U \otimes 1)^{-1}=(1 \otimes U)^{-1} R(\theta)(1 \otimes U),
$$

$U$ being the matrix in $\mathbb{C}^{N}$ of finite order $g$, i.e. $U^{g}=1$.

(iii) Most $R$-matrices (excluding $R$-matrices $[18,19]$, as well as some others) possess a crossing symmetry

$$
R(\theta)=(V \otimes 1)(P R(-\theta-\varrho) P)^{t_{1}}(V \otimes 1)^{-1},
$$

where $V$ is a matrix in $\mathbb{C}^{N}, \varrho$ is some constant, $t_{1}$ means taking the transpose in the first space $\mathbb{C}^{N}$.

(iv) The relation $R(0) \simeq P$ takes place, $P$ being a permutation matrix in $\mathbb{C}^{N} \otimes \mathbb{C}^{N}$

$$
P_{i_{1} j_{1}}^{i_{2} j_{2}}=\delta_{i_{1} j_{2}} \delta_{j_{1} i_{2}}, \quad P(x \otimes y)=y \otimes x ; \quad x, y \in \mathbb{C}^{N} .
$$

It is convenient to choose a normalization in which $R(\theta)$ has a pole at $\theta=0$, i.e.

$$
\left.\operatorname{Res} R(\theta)\right|_{\theta=0} \simeq P \text {. }
$$

It follows from (1.4)-(1.7) that $R(\theta)$ has poles for $\theta=k \pi, \theta=-\varrho+k \pi, k \in \mathbb{Z}$. Equations (1.7) and (1.1) imply

(v) Unitarity

$$
R(\theta) P R(-\theta) P=\Phi(\theta) \cdot E .
$$

$E$ being a unit matrix in $\mathbb{C}^{N} \otimes \mathbb{C}^{N}, \Phi(\theta)$ some (scalar) function of $\theta$.

Note, that the properties (i)-(v) alone [i.e. without Eq. (1.1)], are rather hard restrictions on the form of $R(\theta)$. In [22], matrix functions of the form

$$
R(\theta)=A+P \operatorname{ctg} \theta-P^{t_{1}} \operatorname{ctg}(\theta+\varrho),
$$

where $A$ is independent of $\theta$, satisfying the requirements (i)-(v), were considered. [Relation (1.6) is fulfilled trivially because $U=1, g=1$.] The great bulk of the solutions obtained (though not all of them) proved to automatically satisfy the triangle Eq. (1.1). In this way more than 30 new solutions to triangle equations were constructed in [22]. Comparing this observation with the method [20] for proving Eqs. (1.1) for elliptic $R$-matrices [19] by the Liouville theorem and with the analogous method used in [24] for Eq. (1.3), we found out that the properties (i)-(v) complemented by conditions

(vi) $R(\theta)$ has no poles at $\theta \neq k \pi, \theta \neq-\varrho^{\prime}+k \pi, k \in \mathbb{Z}$,

$$
R^{ \pm}=\lim _{\theta \rightarrow \pm i \infty} R(\theta) ; \quad\left|R^{ \pm}\right|<\infty, \quad R_{12}^{+} R_{13}^{+} R_{23}^{+}=R_{23}^{+} R_{13}^{+} R_{12}^{+}
$$

result in the fulfillment of (1.1). For the proof see [25] and Sect. 4 of this paper.

The concept of automorphicity (ii) for classical and quantum $R$-matrices was introduced in [19] where its connection with automorphisms of the Lie algebra was also established for the case $\mathscr{G}=\operatorname{sl}(N)$. In [24] a rather complete classification of nondegenerate ${ }^{1}$ solutions (1.3) for all simple Lie algebras was made using this idea. In particular, all elliptic and trigonometric $r$-matrices were found. Elliptic $r$-matrices turned out to be connected with the $s l(N)$ algebra and be exhausted by those constructed in [19] where the corresponding quantum $R$-matrix (in the fundamental representation) was also found.

\footnotetext{
${ }^{1}$ An $r$-matrix is called nondegenerate provided $\operatorname{det}\left\|r_{\mu \nu}\right\| \neq 0$, where $r=r_{\mu \nu} E^{\mu} \otimes E^{\nu}, E^{\mu}$ is the basis in $\mathscr{G}$
} 
As for the trigonometric $r$-matrices, constructed in [24], which are not degenerate elliptic ones, their corresponding quantum $R$-matrices were known only in a few particular cases $[15,17]$.

In the present paper, six new infinite series of trigonometric quantum $R$-matrices corresponding to the fundamental representations of the nonexceptional simple Lie algebras $s l(N), s p(N), o(N)$ have been constructed. The technique we applied consisted of constructing $R$-matrices obeying the above-mentioned properties (i) (vi) and possessing the quasi-classical limit (1.2) with the classical $r$-matrices found in $[24]^{2}$. The $R$-matrices constructed are given in two equivalent representations: in an additive one (as a sum of poles with matrix coefficients) and in a multiplicative one (as a ratio of entire matrix functions). From the point of view of the above properties (i)-(iv) these representations complement each other: the additive representation possesses the explicit crossing-symmetry while the multiplicative one possesses the explicit unitarity. The main results of the paper were published briefly in [25-27]. Note that our methods were also generalized to the case of $R$-matrices connected with Lie superalgebras [28, 29].

An alternative approach to the construction of quasiclassical trigonometric $R$-matrices were introduced in [30]. The point of this approach is an interwinding relation for the quantum $L$-operators for generalized periodic Toda lattices. In this way the connection between quasiclassical trigonometric $R$-matrices and quantum Kac-Moody algebras $[10,30]$ is established.

We shall proceed as follows. In Sect. 2 some necessary information from the theory of Lie algebras is presented. In Sect. 3, trigonometric classical $r$-matrices of [24] are considered. In Sect. 4 the corresponding quantum $R$-matrices are constructed. Section 5 contains a discussion of the integrable quantum systems associated with these $R$-matrices: 1 ) two-dimensional generalized Toda lattices; 2 ) anisotropic generalizations of the $s l(N), s p(N)$, and $o(N)$ invariant models of one-dimensional magnetics. In Sect. 6, factorized representations for trigonometric $R$-matrices are obtained. A representation of this type was first obtained in [31] for an elliptic $R$-matrix [19]. It is expressed with the help of the ordered exponent

$$
R(\theta)=P \exp \left\{\frac{1}{2} \int_{\theta-2 \varphi}^{\theta+2 \varphi} r(s) d s\right\},
$$

where $r(\theta)$ is the corresponding classical $r$-matrix, normalized at $\theta=0$ by the condition

$$
r(\theta)=\frac{P-1}{\theta}+\mathrm{const}+O(\theta)
$$

$P$ is the permutation operator (1.6). As it was recognized in [32], Eq. (1.12) may be rewritten in the form

$$
R(\theta)=\sigma(\theta-2 \varphi) \sigma^{-1}(\theta+2 \varphi)
$$

where the function

$$
\sigma(\theta)=P \exp \left\{\frac{1}{2} \int_{\theta}^{\theta_{0}} r(s) d s\right\} \sigma_{0}
$$

\footnotetext{
${ }^{2}$ This technique, however, is not applicable to the $R$-matrices for the non-fundamental representations of $\mathscr{G}$, because these $R$-matrices do not possess the property (vi)
} 
can be interpreted as a matrix generalization of the Weierstrasse elliptic $\sigma$-function $\left[\frac{1}{2} r(s)\right.$ is treated, accordingly, as a matrix generalization of the elliptic $\zeta$-function].

We make use of this analogy to introduce the matrix generalizations of the trigonometric functions, corresponding to the obtained trigonometric $R$-matrices.

\section{Some Information from the Theory of Simple Lie Algebras}

This section presents briefly some necessary information from the theory of simple Lie algebras. For details and proof see [24].

Let $\mathscr{G}=\mathscr{G}(N)$ be a Lie algebra of $N$ by $N$ matrices. In this paper we restrict ourselves to the consideration of the classical matrix Lie algebras $s l(N), \operatorname{sp}(N), o(N)$ in the fundamental representations:

$$
\begin{gathered}
s l(n) \stackrel{\text { def }}{=}\{X \in \operatorname{Mat}(n, \mathbb{C}) \mid \operatorname{Sp} X=0\}, \\
s p(2 n) \stackrel{\text { def }}{=}\left\{X \in \operatorname{Mat}(2 n, \mathbb{C}) \mid X^{t}=-\widetilde{S} X \widetilde{S}^{-1}\right\}, \\
o(n) \stackrel{\text { def }}{=}\left\{X \in \operatorname{Mat}(n, \mathbb{C}) \mid X^{t}=-S X S^{-1}\right\},
\end{gathered}
$$

where $t$ denotes taking the transpose, while the $n$ by $n$ matrix $S$ and the $2 n$ by $2 n$ matrix $\tilde{S}$ are of the form

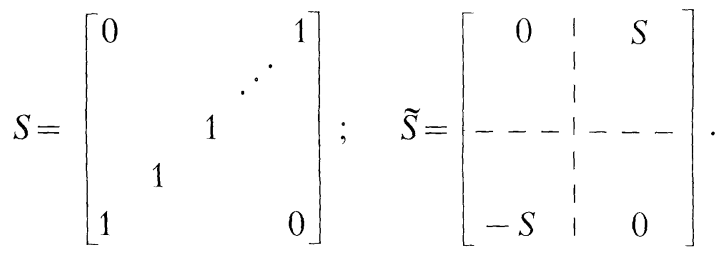

Let $\left\{E_{i}\right\}$ be the basis of generators in $\mathscr{G}(N)$. The scalar product in $\mathscr{G}$ is specified by the Killing form [note, that the definitions (2.1) entail $\operatorname{Sp} X=0$ ]:

$$
(X, Y)=\operatorname{Sp}(X Y) ; \quad X, Y \in \mathscr{G} .
$$

The Killing form has the invariance property

$$
(X,[Y, Z])=([X, Y], Z),
$$

$[$,$] denoting a commutator. The tensor of the Killing form is$

$$
g_{i j}=\left(E_{i}, E_{j}\right)=\operatorname{Sp}\left(E_{i} E_{j}\right) .
$$

In order to describe the trigonometric solutions of Eqs. (1.3), one has to use the concept of the Coxeter automorphism of algebra $\mathscr{G}$ (see, e.g., [24]).

An automorphism of a Lie algebra is a one-to-one linear transformation that preserves the commutation operation. An inner automorphism is a product of a finite number of automorphisms of the form $e^{\text {ad } x}, x \in \mathscr{G}$, with ad $x$ being a linear operator acting by the rule

$$
\operatorname{ad} x(y)=[x, y] ; \quad x, y \in \mathscr{G} .
$$

Let $\mathscr{G}$ be a simple Lie algebra. Any automorphism of $\mathscr{G}$ can be represented as $\varphi_{\text {int }} \cdot \varphi_{\tau}$, where $\varphi_{\text {int }}$ is an inner automorphism and $\varphi_{\tau}$ is induced by the automorphism $\tau$ of the Dynkin diagram of algebra $\mathscr{G}$. 
Let us fix the automorphism $\tau$ of the Dynkin diagram. The automorphism $A_{\mathcal{c}}=\varphi_{\text {int }} \cdot \varphi_{\tau}$ is said to be a Coxeter one provided

1) algebra $\mathscr{G}_{0} \stackrel{\text { def }}{=}\left\{X \in \mathscr{G} \mid A_{c}[X]=X\right\}$ is Abelian,

2) $A_{c}$ has minimum order among the automorphisms $A^{\prime}=\varphi_{\mathrm{int}}^{\prime} \cdot \varphi_{\tau}$ such that the algebra $\mathscr{G}_{0}^{\prime}$ is abelian.

In the following the pair of the algebra and its Coxeter automorphisms will be denoted by the symbol $\widetilde{\mathscr{G}}=\left(\mathscr{G}, A_{c}\right)$. For classical Lie algebras $A_{n-1}=s l(n)$, $B_{n}=o(2 n+1), C_{n}=s p(2 n), D_{n}=o(2 n)$ there are seven infinite series of pairs: $\widetilde{\mathscr{G}}^{n}=A_{n-1}^{(1)}, A_{2 n}^{(2)}, A_{2 n-1}^{(2)}, B_{n}^{(1)}, C_{n}^{(1)}, D_{n}^{(1)}, D_{n}^{(2)}$. In these notations, the numbers in parentheses show the order of the automorphism $\tau$ of the Dynkin diagram.

The explicit form of the Coxeter automorphisms for the algebras (2.1) under consideration is given in Table 1 borrowed from the paper [24]. The notations: $h$ is the order of an automorphism, $A_{c}[X]$ is the image of an element $X \in \mathscr{G}$ under the action of an automorphism, $\omega=\exp (2 \pi i / h), \sigma_{x}$ in the definition of the matrix $T$ for the series, $D_{n}^{(2)}$ denotes a diagonal 2 by 2 block with the elements $\sigma_{11}=\sigma_{22}=0$; $\sigma_{12}=\sigma_{21}=1$.

The eigenvalues of $A_{c}$ equal $\omega^{j}, j \in \mathbb{Z}$. Therefore, $\mathscr{G}$ may be represented in the form

$$
\mathscr{G}=\sum_{j=0}^{h-1} \mathscr{G}_{j} ; \quad \mathscr{G}_{j} \stackrel{\text { def }}{=}\left\{X \in \mathscr{G} \mid A_{c}[X]=\omega^{j} X\right\} .
$$

Note that by the definition of a Coxeter automorphism the algebra $\mathscr{G}_{0}$ is abelian.

Let $r=\operatorname{dim} \mathscr{G}_{0} . \quad$ There exist elements $e_{0}, \ldots, e_{r} \in \mathscr{G}_{1} ; f_{0}, \ldots, f_{r} \in \mathscr{G}_{-1}$; $\tilde{h}_{0}, \ldots, \tilde{h}_{r-1} \in \mathscr{G}_{0}$, such that

1) $e_{0}, \ldots, e_{r}$ form the basis in $\mathscr{G}_{1} ; f_{0}, \ldots, f_{r}$ form one in $\mathscr{G}_{-1} ; \tilde{h}_{0}, \ldots, \tilde{h}_{r-1}$ form the basis in $\mathscr{G}_{0}$, normalized by the condition

$$
\left(\tilde{h}_{i}, \tilde{h}_{j}\right)=2 \delta_{i j}
$$

2) The following relations are satisfied:

$$
\begin{gathered}
{\left[\tilde{h}_{a}, \tilde{h}_{b}\right]=0,} \\
{\left[\tilde{h}_{a}, e_{i}\right]=\alpha_{i}^{a} e_{i}, \quad\left[\tilde{h}_{a}, f_{i}\right]=-\alpha_{i}^{a} f_{i},} \\
{\left[e_{i}, f_{j}\right]=2 \delta_{i j} \frac{\left(\alpha_{i}, \tilde{h}\right)}{\left(\alpha_{i}, \alpha_{i}\right)},}
\end{gathered}
$$

Table 1. Coxeter automorphisms of the classical Lie algebras

\begin{tabular}{lllll}
\hline$\tilde{\mathscr{G}}$ & $\mathscr{G}(N)$ & $h$ & $A[X]$ & $T$ \\
\hline $\mathrm{A}_{n-1}^{(1)}$ & $\operatorname{sl}(n)$ & $n$ & $T X T^{-1}$ & $\omega^{(n-1) / 2} \operatorname{diag}\left(1, \omega^{-1}, \omega^{-2}, \ldots, \omega^{1-n}\right)$ \\
$A_{2 n}^{(2)}$ & $s l(2 n+1)$ & $4 n+2$ & $-T X^{t} T^{-1}$ & $S \operatorname{diag}\left(1, \xi, \ldots, \xi^{2 n}\right) ; \xi=-\omega$ \\
$A_{2 n-1}^{(2)}$ & $s l(2 n)$ & $4 n-2$ & $-T X^{t} T^{-1}$ & $S \operatorname{diag}\left(1, \xi, \ldots, \xi^{n-2}, \xi^{n-1}, \xi^{n-1}, \xi^{n}, \ldots, \xi^{2 n-2}\right) ; \xi=-\omega$ \\
$C_{n}^{(1)}$ & $s p(2 n)$ & $2 n$ & $T X T^{-1}$ & $\omega^{(2 n-1) / 2} \operatorname{diag}\left(1, \omega^{-1}, \ldots, \omega^{1-2 n}\right)$ \\
$B_{n}^{(1)}$ & $o(2 n+1)$ & $2 n$ & $T X T^{-1}$ & $\operatorname{diag}\left(1, \omega^{-1}, \ldots, \omega^{1-2 n}, 1\right)$ \\
$D_{n}^{(1)}$ & $o(2 n)$ & $2 n-2$ & $T X T^{-1}$ & $\operatorname{diag}\left(1, \omega^{-1}, \ldots, \omega^{2-n}, \omega^{1-n}, \omega^{1-n}, \omega^{-n}, \ldots, \omega^{3-2 n}, 1\right)$ \\
$D_{n}^{(2)}$ & $o(2 n)$ & $2 n$ & $T X T^{-1}$ & $\operatorname{diag}\left(\omega^{-1}, \omega^{-2}, \ldots, \omega^{1-n}, \sigma_{x}, \omega^{-n-1}, \ldots, \omega^{1-2 n}\right)$ \\
\hline
\end{tabular}


with

$$
\left(\alpha_{i}, \alpha_{i}\right)=\sum_{a=1}^{r-1} \alpha_{i}^{a} \alpha_{i}^{a}, \quad\left(\alpha_{i}, \tilde{h}\right)=\sum_{a=1}^{r-1} \alpha_{i}^{a} \tilde{h}_{a}
$$

The $r$-dimensional vectors $\alpha_{j}=\left(\alpha_{j}^{0}, \ldots, \alpha_{j}^{r-1}\right)$ are called simple weights for the $\left(\mathscr{G}, A_{c}\right)$ pair. The explicit realizations for the generators $\tilde{h}_{a}, e_{i}, f_{i}$ and for the systems of simple weights are presented in Appendix A.

The matrix

$$
t=\lambda \sum g^{i j} E_{i} \otimes E_{j}
$$

plays in important role in describing the solutions of the triangle equations. Here we choose $\lambda=1$ for $A_{n-1}^{(1)}$ and $\lambda=2$ for other series; tensor $g^{i j}$ is inverse to tensor of the Killing form (2.4). It follows from the invariance of the Killing form (2.3) that for any $X \in \mathscr{G}$,

$$
[t, X \otimes 1+1 \otimes X]=0 .
$$

As may be easily checked, $(A \otimes A)[t]=t$. Hence, $t$ may be represented as

$$
\begin{aligned}
t & =\sum_{j=0}^{h-1} t_{j} ; \quad t_{j} \in \mathscr{G}_{j} \otimes \mathscr{G}_{-j}, \\
t_{j} & =h^{-1} \sum_{n=0}^{h-1} \omega^{-n j}\left(A_{c} \otimes 1\right)^{n}[t],
\end{aligned}
$$

$h$ being the order of $A_{c}$. Note, (2.7), (2.12), (2.14) entail that

$$
t_{0}=\frac{\lambda}{2} \sum_{a} \tilde{h}_{a} \otimes \tilde{h}_{a}
$$

\section{Classical Triangle Equations}

The most complete results for the solutions of the classical triangle Eq. (1.3) were obtained in [24]. Below we present some necessary information from this work.

\subsection{Classical $r$-Matrices}

As has been noted in the Introduction, Eq. (1.3) is written in terms of commutators. Therefore, one can assume $r(\theta) \in \mathscr{G}(N) \otimes \mathscr{G}(N), \mathscr{G}=\mathscr{G}(N)$ being a Lie algebra of $N$ by $N$ matrices.

For any Lie algebra, there is a simplest rational solution to Eq. (1.3) of the form $[19,23]$,

$$
r(\theta)=\frac{t}{\theta}
$$

For these solutions, Eq. (1.3) reduces to relation (2.13). The trigonometric solutions for Eq. (1.3) are obtained [24] via "averaging" (the term introduced in [33]) the elementary pole (3.1) over the one-dimensional lattice $\theta=k \pi / \mu, k \in \mathbb{Z}$, using the Coxeter automorphism $A_{c}$ of the algebra $\mathscr{G}$

$$
r(\theta)=h^{-1} \sum_{j=0}^{h-1} A_{c}^{j}[t] \operatorname{ctg}((\theta \mu-k \pi) / h)=i t_{0}+2 i \sum_{j=0}^{h-1} \frac{\exp (2 i \theta \mu j / h) t_{j}}{\exp (2 i \mu \theta)-1},
$$


where $t_{j}$ are defined in (2.14), $\mu$ is a constant. Thus, the trigonometric solutions are determined by the choice of the pair $\widetilde{G}=\left(\mathscr{G}, A_{c}\right)$. Remember, that for classical Lie algebras there are six infinite series of pairs listed in Table 1. Formula (3.2) was obtained in [24] for the generic case, and in [34, 35] for the series $A_{n-1}^{(1)}, B_{n}^{(1)}, C_{n}^{(1)}, D_{n}^{(1)}$. Note that the function $r(\theta)$ is unambiguously determined by the following three properties:

1) $r(\theta)$ is meromorphic in $\theta$ with the set of poles $\theta=k \pi / \mu, k \in \mathbb{Z}$, and at zero it has the residue $t$,

$$
\left.\operatorname{Res}(\theta)\right|_{\theta=0} \simeq t
$$

2) Automorphicity (quasi-periodicity)

$$
r(\theta+\pi)=\left(A_{c} \otimes 1\right)[r(\theta)]=\left(1 \otimes A_{c}\right)^{-1}[r(\theta)] .
$$

3) Asymptotic behavior

$$
\left.r(\theta)\right|_{\theta \rightarrow \pm i \infty}=\mp i t_{0},
$$

where $t_{0}$ is given in (2.14). With the help of Eqs. (2.14) and (3.2) one can easily derive an additional property of $r(\theta)$ :

4) Classical unitarity

$$
r(\theta)=-\operatorname{Pr}(-\theta) P,
$$

$P$ being the permutation matrix (1.6).

Reference [24] contained a highly simple and elegant proof of the fact that $r(\theta)$ satisfies the classical triangle Eq. (1.3). This proof will be given below. It is based on the abovesaid properties 1)-4) of the function $r(\theta)$. Let us denote by $\psi\left(\theta, \theta^{\prime}\right)$ the lefthand side of Eq. (1.3) and consider it as a function of $\theta$, keeping $\theta^{\prime}$ fixed. By virtue of (3.3), (3.4) it is quasi-periodic,

$$
\Psi\left(\theta+\pi g / \mu, \theta^{\prime}\right)=\left(A_{c} \otimes 1 \otimes 1\right)\left[\Psi\left(\theta, \theta^{\prime}\right)\right]
$$

and has simple poles at $\theta=k \pi, \theta=-\theta^{\prime}+k \pi, k \in \mathbb{Z}$. Using relations (2.13), (3.3), (3.6), (3.7), one can readily exhibit that the residues in these poles vanish. It follows from (3.5) that $\psi\left(\theta, \theta^{\prime}\right)$ is finite when $\theta \rightarrow \pm i \infty$. Together with the periodicity of $\psi\left(\theta, \theta^{\prime}\right)$ in $\theta$ (with the period $g \pi / \mu$ ) this means that $\psi\left(\theta, \theta^{\prime}\right)$ is independent of $\theta$. Similarly, we can show $\psi\left(\theta, \theta^{\prime}\right)$ to be independent of $\theta^{\prime}$. Now, tending $\theta \rightarrow i \infty$, and then $\theta^{\prime} \rightarrow i \infty$, using (3.5) and remembering that $t_{0} \in \mathscr{G}_{0} \otimes \mathscr{G}_{0}$, where $\mathscr{G}_{0}$ is an Abelian subalgebra of $\mathscr{G}$, one finds $\psi\left(\theta, \theta^{\prime}\right) \equiv 0$.

In our next section we shall construct the quantum $R$-matrices, corresponding to the classical $r$-matrices (3.2), for the fundamental representations of the algebras (2.1) in question. In this connection, it is useful to rewrite relation (3.2) taking into account the explicit form of $t$ for the representations (2.1). Choosing some particular basis in $\mathscr{G}$, it is easy to show that ${ }^{3}$

$$
\begin{gathered}
t_{s l(N)}=\lambda P, \\
t_{s p(N)}=P-\tilde{S} P^{t_{1}} \tilde{S}^{-1}, \\
t_{o(N)}=P-S P^{t_{1}} S^{-1},
\end{gathered}
$$

\footnotetext{
${ }^{3}$ In the case of $s l(N)$ algebra we drop in (3.8) a term, proportional to unity in $\mathbb{C}^{N} \otimes \mathbb{C}^{N}$, which does not affect Eq. (1.3)
} 
where $\lambda$ was defined after Eq. (2.12), $P$ is the permutation matrix (1.6), $t_{1}$ means taking the transpose in the first space $\mathbb{C}^{N}$. Here and after we use boldface letters to denote products of the form $A \otimes 1$, e.g.

$$
\mathbf{S}=S \otimes 1, \quad \widetilde{\mathbf{S}}=\tilde{S} \otimes 1, \quad \mathbf{U}=U \otimes 1,
$$

where $\widetilde{S}, S$ were defined in (2.2).

Let $U$ be a matrix in $\mathbb{C}^{N}$ of a finite order $g, U^{g}=1$. Introduce the function

$$
Z(\theta)=Z(\theta, P)=g^{-1} \sum_{k=0}^{g-1} \mathbf{U}^{k} P \mathbf{U}^{-k} \operatorname{ctg}((\theta-k \pi) / g)
$$

It is easy to check that $Z(\theta)$ obeys the relations

$$
\begin{gathered}
\left.\operatorname{Res} Z(\theta)\right|_{\theta=0}=P, \\
Z(\theta+\pi)=\mathbf{U} Z(\theta) \mathbf{U}^{-1}, \quad Z(-\theta)=-P Z(\theta) P .
\end{gathered}
$$

Let us now choose in Eq. (3.2) $\mu=2$ for the series $A_{2 n}^{(2)}, A_{2 n-1}^{(2)}$ and $\mu=1$ for the other series. Using (3.8)-(3.12) and the expression for $A[X]$ from Table 1, we can represent the function $r(\theta)$ in the form

$$
\begin{gathered}
r(\theta)=Z(\theta), \quad \tilde{\mathscr{G}}=A_{n-1}^{(1)}, \\
r(\theta)=Z(\theta)-(B \otimes 1) Z^{t_{1}}(\theta-\Delta)(B \otimes 1)^{-1}, \widetilde{\mathscr{G}} \neq A_{n-1}^{(1)} .
\end{gathered}
$$

The values of $g$ and the matrices $U$ and $B$ for each series are presented in Table $2, t_{1}$ denotes taking the transpose in the first space $\mathbb{C}^{N}$

$$
\Delta= \begin{cases}0, & \tilde{G}=B_{n}^{(1)}, C_{n}^{(1)}, D_{n}^{(1)}, D_{n}^{(2)}, \\ \pi / 2 & \tilde{G}=A_{2 n}^{(2)}, A_{2 n-1}^{(2)} .\end{cases}
$$

Note, that the matrix $U$ possesses the property

$$
B U B^{-1}=U^{-1} \text {. }
$$

Let us give one more useful formula resulting from (2.14) and (3.8):

$$
t_{0}= \begin{cases}\widetilde{P}, & \tilde{G}=A_{n-1}^{(1)}, \\ \widetilde{P}-\tilde{K}, & \widetilde{G} \neq A_{n-1}^{(1)},\end{cases}
$$

Table 2. The parameters of the solutions of triangle equations. The quantities $h, T$ for each series are given in Table $1 . S, \tilde{S}$ are defined by Eqs. (2.2)

\begin{tabular}{llllll}
\hline Ser. & $g$ & $B$ & $U$ & $\varrho$ & $\left\{c_{a}\right\}, a=0, \ldots, n-1$ \\
\hline $\mathrm{A}_{n-1}^{(1)}$ & $h$ & - & $T$ & & - \\
$A_{2 n}^{(2)}$ & $h / 2$ & $T$ & $T\left(T^{t}\right)^{-1}$ & $N \varphi--\frac{\pi}{2}$ & $c_{a}=-1$ \\
$A_{2 n-1}^{(2)}$ & $h / 2$ & $T$ & $T\left(T^{t}\right)^{-1}$ & $N \varphi-\frac{\pi}{2}$ & $c_{a}=2(a-n+1) /(2 n-1)$ \\
$C_{n}^{(1)}$ & $h$ & $\tilde{S}$ & $T$ & $(N+2) \varphi$ & $c_{a}=(2 a-n+1) / n$ \\
$B_{n}^{(1)}$ & $h$ & $S$ & $T$ & $(N-2) \varphi$ & $c_{a}=-a / n$ \\
$D_{n}^{(1)}$ & $h$ & $S$ & $T$ & $(N-2) \varphi$ & $c_{a}=0$ \\
$D_{n}^{(2)}$ & $h$ & $S$ & $T$ & $(N-2) \varphi$ & $c_{n-1}=0 ; c_{a}=-(2 a-n+2) / n, a<n-1$ \\
\hline
\end{tabular}


where

$$
\begin{gathered}
\widetilde{P}=g^{-1} \sum_{k=0}^{g-1} \mathbf{U}^{k} P \mathbf{U}^{-k}, \\
\tilde{K}=g^{-1} \sum_{k=0}^{g-1} \mathbf{U}^{k} K \mathbf{U}^{-k}, \\
K=\mathbf{B} P^{t_{1}} \mathbf{B}^{-1}, \quad K^{2}=N K, \\
\left(P^{t_{1}}\right)_{i_{1} i_{2}}^{j_{1} j_{2}}=\delta_{i_{1} i_{2}} \delta_{j_{1} j_{2}} .
\end{gathered}
$$

Here $N$ is the matrix dimension of $\mathscr{G}$.

\subsection{Classical Toda Lattices}

In this subsection we consider a class of integrable classical systems related to the $r$-matrices (3.2), called by the two-dimensional generalized periodic Toda lattices $[36,37]$.

Consider the pair $\widetilde{G}=\left(\mathscr{G}, A_{c}\right)$ where $\mathscr{G}$ is a simple Lie algebra and $A_{c}$ is its Coxeter automorphism.

The system with the Lagrangian

$$
\begin{gathered}
\mathscr{L}=\gamma^{-1} \int d x\left[\frac{1}{2}\left(\partial_{\mu} u\right)^{2}-V(u)\right], \\
V(u)=m^{2} \sum_{i=0}^{r} \frac{2}{\left(\alpha_{i}, \alpha_{i}\right)} e^{2\left(\alpha_{i}, u\right)}, \\
\left(\alpha_{i}, u\right)=\sum_{a=0}^{r-1} \alpha_{i}^{a} u_{a},
\end{gathered}
$$

is called a two-dimensional Toda lattice associated with $\tilde{G}$. Here $u_{a}=u_{a}(x, t)$, $a=0, \ldots, r-1$, is $a$ set of scalar fields in two-dimensional space-time, $r=\operatorname{dim} \mathscr{G}_{0}$, $\left\{\alpha_{j}\right\}$ is a system of simple weights $\tilde{\mathscr{G}}$ [see Eqs. (2.7)-(2.11)]. The equations of motion

$$
\left(\partial_{t}^{2}-\partial_{x}^{2}\right) u_{a}=-\frac{\partial}{\partial u_{a}} V(u)
$$

can be represented in Zakharov-Shabat's form

$$
\begin{gathered}
\partial_{t} L-\partial_{x} M+[L, M]=0, \\
L(\theta)=\partial_{t} u+\lambda m e^{\mathrm{ad} u} I_{+}+\lambda^{-1} m e^{-\mathrm{ad} u} I_{-}, \\
M(\theta)=\partial_{x} u-\lambda m e^{\mathrm{ad} u} I_{+}+\lambda^{-1} m e^{-\mathrm{ad} u} I_{-}, \\
u=\sum_{a=0}^{r-1} u^{a} \tilde{h}_{a}, \quad \lambda=\exp (-2 i \theta / g), \\
I_{+}=\sum_{i=0}^{r} e_{i}, \quad I_{-}=\sum_{i=0}^{r} f_{i} .
\end{gathered}
$$

The operator ad $u$ was defined by (2.5), the values of $g$ can be found in Table 2.

The canonical Poisson bracket has the form

$$
\left\{\pi_{a}(x), u_{b}(y)\right\}=\delta(x-y) \delta_{a b},
$$

with $\pi=\gamma^{-1} \partial_{t} u$. 
The most efficient approach to integrable systems with an ultralocal (i.e. having no derivatives of $\delta$-function) Poisson bracket is the $r$-matrix technique [11]. It is based on the fact that a Poisson bracket of two L-operators can be written in the so-called $r$-matrix form:

$$
\left\{L(\theta, x) \otimes L\left(\theta, x^{\prime}\right)\right\}=i \gamma \delta\left(x-x^{\prime}\right)\left[L(\theta) \otimes 1+1 \otimes L\left(\theta^{\prime}\right), r\left(\theta^{\prime}-\theta\right)\right],
$$

where $r(\theta)$ is a classical $r$-matrix.

For the $L$-operator (3.25) and $r$-matrix (3.13), (3.14) this relation was proved in [24]. We will not present the proof here since relation (3.30) can be treated as a quasi-classical limit of the relation (5.4) for the corresponding quantum L-operator, discussed in Sect. 5.

\section{Quantum Triangle Equations}

In the present section we shall construct quantum $R$-matrices, corresponding to the classical $r$-matrices (3.14), for the series $A_{2 n}^{(2)}, A_{2 n-1}^{(2)}, B_{n}^{(1)}, C_{n}^{(1)}, D_{n}^{(1)}, B_{n}^{(2)}$. The quantum $R$-matrix for the series $A_{n}^{(1)}$ was already known; it will be given in the end of this section. As has been pointed out in the Introduction, quantum $R$-matrices depend essentially on the representation of algebra $\mathscr{G}$. In the present paper we limit ourselves to the consideration of fundamental representations of $\mathscr{G}$, defined in (2.1).

\subsection{A Simple Theorem}

The basic idea of our approach is to employ the following theorem [25].

Let $R(\theta)$ be a meromorphic function of $\theta$ with the following properties

(i) Automorphicity (quasi-periodicity) and invariance:

$$
R(\theta+\pi)=(U \otimes 1) R(\theta)(U \otimes 1)^{-1}=(1 \otimes U)^{-1} R(\theta)(1 \otimes U),
$$

where $U$ is the matrix in $\mathbb{C}^{N}$ of finite order g, i.e. $U^{g}=1$. It follows from (4.1) that $R(\theta)$ is periodic with the period $g \pi$.

(ii) Crossing symmetry:

$$
R(\theta)=(V \otimes 1)(P R(-\theta-\varrho) P)^{t_{1}}(V \otimes 1)^{-1} .
$$

Here $\varrho$ is a constant, $\varrho \neq 0 ; V$ is a matrix in $\mathbb{C}^{N}, t_{1}$ denotes taking the transpose with respect to the first space $\mathbb{C}^{N}, P$ is the permutation matrix (1.6).

(iii) Unitarity:

$$
R(\theta) P R(-\theta) P=E \Phi(\theta),
$$

with $E$ being a unit matrix in $\mathbb{C}^{N} \otimes \mathbb{C}^{N}$, while $\Phi(\theta)$ is some scalar function of $\theta$.

(iv) Asymptotic behavior:

$$
\begin{gathered}
R(\theta) \text { is finite when } \theta \rightarrow \pm i \infty \\
R_{12}^{+} R_{13}^{+} R_{23}^{+}=R_{23}^{+} R_{13}^{+} R_{12}^{+} ; \quad R^{+}=\lim _{\theta \rightarrow \pm i \infty} R(\theta) .
\end{gathered}
$$

(v) Pole structure: $R(\theta)$ has simple poles, which are located at $\theta=k \pi$, $\theta=-\varrho+k \pi, k \in \mathbb{Z}$, only. The residue of $R(\theta)$ for $\theta=0$ is proportional to $P$ :

$$
\left.\operatorname{Res} R(\theta)\right|_{\theta=0} \simeq P .
$$

The remaining residues are fixed by the properties (i)-(ii). 
Theorem. It follows from (i)-(v) that $R(\theta)$ satisfies Eq. (1.1).

Proof. Let us denote by $\psi\left(\theta, \theta^{\prime}\right)$ the difference between the right- and left-hand sides of Eq. (1.1) and consider $\psi\left(\theta, \theta^{\prime}\right)$ as a function of $\theta$ keeping $\theta^{\prime}$ fixed. It follows from (i), (v), that $\psi\left(\theta, \theta^{\prime}\right)$, similarly to $R(\theta)$, possesses the automorphicity property, is periodic with the period $g \pi$ and has poles at $\theta=k \pi, \theta=-\varrho+k \pi, \theta=-\theta^{\prime}+k \pi$, $\theta=-\theta^{\prime}-\varrho+k \pi, k \in \mathbb{Z}$. Using (ii), (iii), and (v), one can show that the residues at these poles vanish. (Note that due to automorphicity it is sufficient to consider only four poles.) Next, Eq. (4.4) entails that $\psi\left(\theta, \theta^{\prime}\right)$ is finite for $\theta \rightarrow \pm i \infty$. This means [provided periodicity of $\psi\left(\theta, \theta^{\prime}\right)$ is taken into account] that $\psi\left(\theta, \theta^{\prime}\right)$ is a constant, i.e. independent of $\theta$. Similarly $\psi\left(\theta, \theta^{\prime}\right)$ may be proved to be independent of $\theta^{\prime}$. Letting now $\theta \rightarrow i \infty$ and then $\theta^{\prime} \rightarrow i \infty$ and using Eq. (4.5), we obtain $\Psi\left(\theta, \theta^{\prime}\right) \equiv 0$. Q.E.D.

Remark 1. It is not difficult to obtain a generalization of the above theorem to the elliptic case. For $R$-matrices with one series of poles [19] [i.e. having no property (ii)] the theorem was used in [20].

Remark 2 . The property of $R$-matrix to be quasi-classical has not been used in the proof. Therefore, the theorem can be applied to nonquasi-classical $R$-matrices as well, in particular to those of [22] (the corresponding calculations were performed by $\mathrm{O}$. Vasiliev).

It would also be interesting to investigate new $R$-matrices, found in [38], from the point of view of the above theorem.

\subsection{Quantization of Trigonometric r-Matrices}

Below we construct a quantum $R$-matrix $R(\theta, \varphi)$ which obeys the conditions of the theorem of the previous subsection and has the quasi-classical limit (1.2) with the classical $r$-matrix (3.14).

An analysis of known $R$-matrices for the cases $A_{2}^{(2)}[17]$ and $C_{1}^{(1)}[15]$ shows that they may be represented in the form

$$
\begin{gathered}
R(\theta, \varphi)=R_{0}(\varphi)+\sin 2 \varphi\left[Z(\theta)-\mathbf{C}(\varphi) \mathbf{B} Z^{t_{1}}(\theta+\varrho)(\mathbf{C}(\varphi) \mathbf{B})^{-1}\right], \\
R_{0}(\varphi)=1+(\cos 2 \varphi-1) t_{0}^{2}, \\
\mathbf{C}(\varphi)=C(\varphi) \otimes 1, \quad \mathbf{B}=B \otimes 1, \quad \mathbf{U}=U \otimes 1,
\end{gathered}
$$

where $t_{0}$ is given in (3.17), the other notations are defined by Eqs. (2.14), (3.10), (3.14) and Table $1, C(\varphi)$ is a diagonal matrix in $\mathbb{C}^{N}$, such that

$$
\begin{gathered}
{[C(\varphi), U]=[C(\varphi), \tilde{K}]=0,} \\
C(\varphi) B=B C^{-1}(\varphi),
\end{gathered}
$$

where $\widetilde{K}$ is defined in $(3.19)^{4}$. It is convenient to write $C(\varphi)$ in the form

$$
C(\varphi)=\exp (i \varphi(c, \tilde{h})) ; \quad(x, y)=\sum_{a=0}^{r-1} x_{a} y_{a},
$$

\footnotetext{
${ }^{4}$ Condition (4.11) eliminates gauge freedom in choosing $C(\varphi)$ connected with the transformations $R \rightarrow(D \otimes D) R(D \otimes D)$, where $D$ is a diagonal matrix commuting with the matrix $\mathrm{B}$
} 
where $\left\{\tilde{h}_{a}\right\}$ is the basis in $\mathscr{G}_{0}, r=\operatorname{dim} \mathscr{G}_{0}$. Note that in our realization of $\mathscr{G}$,

$$
\tilde{h}_{a}=e_{a, a}-e_{N-a-1 . N-a-1} ; \quad\left(e_{i j}\right)_{\alpha \beta}=\delta_{i \alpha} \delta_{j \beta},
$$

$N$ being the matrix dimension of $\mathscr{G}$ (see Sect. 2 and Appendix A).

It seems very reasonable to use Ansatz (4.7)-(4.12) in a general case. Indeed, with the help of relations (3.11), (3.17), (4.8) it is easy to show that $R(\theta, \varphi)$, so defined, satisfies conditions (i), (ii), (v) of the previous subsection. Note that in (4.2) $V=C(\varphi) B$. Next, it follows from the definition (2.14) and the explicit form of Coxeter automorphisms (Table 1), that $t_{0}$ is a diagonal matrix and

$$
t_{0}^{2+k}=t_{0}^{k} ; \quad k>1
$$

Exploiting (3.10), (3.17), (4.7)-(4.9), (4.14) we get

$$
\left.R(\theta, \varphi)\right|_{\theta \rightarrow \pm i \infty}=R^{ \pm}+O\left(e^{ \pm 2 i \theta / g}\right), \quad R^{ \pm}=e^{\mp 2 i \varphi t_{0}} .
$$

Since $t_{0} \in \mathscr{G}_{0} \otimes \mathscr{G}_{0}, \mathscr{G}_{0}$ being an abelian algebra, relation (4.5) is satisfied trivially.

Thus, conditions (i), (ii), (iv), (v) are checked and it is left to check the unitarity condition (4.3). The substitution of (4.7) into (4.3) leads to a system of equations for $C(\varphi)$ and $\varrho$, which allows us to define $C(\varphi)$ and $\varrho$ unambiguously provided (4.11) is taken into account. The required calculations are simple, but tedious. The result is given in the author's work [25] ${ }^{5}$ and we reproduce it in Table 2 . Fortunately there exists a more simple method to prove unitarity for $R$-matrix (4.7), using the quantum $L$-operator for generalized periodic Toda lattices. This proof will be given in Sect. 5. The most essential point of the proof is that $\varrho$ and $C(\varphi)$ from Table 2 are determined unambiguously by the system of equations

$$
-\left(\alpha_{j}, \alpha_{j}\right)+\left(\alpha_{j}, c\right)+\frac{2(\varrho+\Delta \varphi)}{g \varphi}=0 ; \quad j=0,1, \ldots, r,
$$

where $\alpha_{j}^{a}, j=0, \ldots, r$, is the system of simple weights of ( $\left.\mathscr{G}, A_{c}\right)$ (see, Sect. 2), $\Delta$ is defined by (3.15).

Assuming now that the unitarity condition is proved, let us calculate the function $\Phi(\theta)$ from the left-hand side of Eq. (4.3). Denote by $\hat{\Phi}(\theta)$ the left-hand side of (4.3). $\hat{\Phi}(\theta)$ is a meromorphic function of $\theta$ and may have the poles at $\theta=k \pi$, $\theta= \pm \varrho+k \pi, k \in \mathbb{Z}$. By means of (4.1), (4.6), (4.15) it is easy to show that

a) $\hat{\Phi}(\theta+\pi)=(U \otimes 1) \hat{\Phi}(\theta)(U \otimes 1)^{-1}, \quad \lim _{\theta \rightarrow \pm i \infty} \hat{\Phi}(\theta)=E$,

where $E$ is a unit matrix in $\mathbb{C}^{N} \otimes \mathbb{C}^{N}$.

b) When $\theta=k \pi, k \in \mathbb{Z}, \widehat{\Phi}(\theta)$ has the second order poles

$$
\hat{\Phi}(\theta)=-\frac{\sin ^{2} 2 \varphi \cdot E}{(\theta-k \pi)^{2}}+O(1)+O((\theta-k \pi)) .
$$

Next, at $\theta=-\varrho+k \pi, k \in \mathbb{Z}$, the residues of $R(\theta)$ are degenerate matrices

$$
R_{k}=\left.\operatorname{Res} R(\theta)\right|_{\theta=-\varrho+k \pi}=\mathbf{U}^{k} \mathbf{C}(\varphi) K \mathbf{C}^{-1}(\varphi) \mathbf{U}^{-k},
$$

\footnotetext{
${ }^{5}$ In the present paper we use slightly modified (but, of course, equivalent) definitions of Coxeter automorphisms (cf. Table 1 and Table 2 form [25] and present paper)
} 
where the notations (3.20) and (4.9) are used. Since $\operatorname{det} K=0$,

$$
\operatorname{det} R_{k}=0
$$

and consequently,

$$
\operatorname{det}\left(\left.\operatorname{Res} \hat{\Phi}(\theta)\right|_{\theta=\mp \varrho+k \pi}\right)=0 .
$$

The only way to make (4.21) agree with the matrix structure of the left-hand side of (4.3) is to require

c) $\left.\operatorname{Res} \hat{\Phi}(\theta)\right|_{\theta=\mp \varrho+k \pi}=0$.

Now, using the Liouville theorem, we can recover the function $\Phi(\theta)$ unambiguously by the properties (a)-(c) given above,

$$
\hat{\Phi}(\theta)=R(\theta) P R(-\theta) P=\left(1-\frac{\sin 2 \varphi}{\sin ^{2} \theta}\right) E .
$$

Thus, the quantum $R$-matrix for the series $A_{2 n}^{(2)}, A_{2 n-1}^{(2)}, B_{n}^{(1)}, C_{n}^{(1)}, D_{n}^{(1)}, B_{n}^{(2)}$, defined by (4.7) and by the values of $C(\varphi)$ and $\varrho$ from Table 2, satisfies all the conditions of the theorem from the previous subsection and, hence, satisfy Eq. (1.1) as well. In the quasi-classical limit (1.2), Eq. (4.7) yields the corresponding classical $r$-matrix (3.14).

For $\widetilde{\mathscr{G}}=A_{n-1}^{(1)}$ the quantum $R$-matrix has been known before [18]. In our notations it looks like

$$
R(\theta, \varphi)=R_{0}+\sin 2 \varphi Z(\theta),
$$

where $Z(\theta), R_{0}$ was defined by (3.10), (4.8) respectively and the matrix $U$ entering the definition of $Z(\theta)$ was given in Table 2. Clearly, (4.30) has a correct quasiclassical limit with the classical $r$-matrix (3.13).

To conclude this section, we note that the $R$-matrices (4.7), (4.24) have the PTsymmetry and invariance properties

$$
\begin{gathered}
R^{t_{1} t_{2}}(\theta, \varphi)=P R(\theta, \varphi) P, \\
(X \otimes X) R(\theta)(X \otimes X)^{-1}=R(\theta),
\end{gathered}
$$

where $t_{1} t_{2}$ denotes taking the transpose in $\mathbb{C}^{N} \otimes \mathbb{C}^{N}$, and $X$ is any diagonal matrix obeying the relation

$$
B X B^{-1}=X^{-1}
$$

\section{Integrable Quantum Systems}

\subsection{Quantum Toda Lattices}

In this section the quantum variant of generalized periodic Toda lattices will be discussed. For these models we construct the quantum L-operators [26, 30, 39] satisfying relation (5.5). Apparently, this means that they can be integrated by the quantum inverse scattering technique $[8,9]$.

For the series $\widetilde{G}=A_{n-1}^{(1)}$ this problem was solved in [40] (see also $[41,42]$ ). We shall handle the other series from Tables 1,2. 
In the quantum case the Poisson bracket (3.29) is replaced by the commutator

$$
\left[\pi_{a}(x), u_{b}(y)\right]=-i \delta_{a b} \delta(x-y),
$$

where $\pi_{a}^{-1}=\gamma \partial_{t} u_{a}, \gamma=2 \varphi$.

The ultraviolet regularization is achieved by introducing a spatial lattice with a (small) spacing $\delta$. Let us define the variables

$$
\begin{gathered}
u_{a}(n)=\delta^{-1} \int_{x_{n}}^{n_{n}+\delta} u(y) d y, \quad p_{a}(n)=\frac{1}{2} \int_{x_{n}}^{x_{n}+\delta} \partial_{t} u(y) d y, \\
{\left[p_{a}(n), u_{b}\left(n^{\prime}\right)\right]=-i \varphi \delta_{a b} \delta_{n n^{\prime}} .}
\end{gathered}
$$

To apply the quantum inverse problem method $[8,9]$, it is necessary to construct a quantum $L$-operator $\hat{L}(\theta, p, u)$, satisfying the relation

$$
\begin{gathered}
L_{n}^{1}(\theta) L_{n}^{2}\left(\theta^{\prime}\right) R^{12}\left(\theta^{\prime}-\theta\right)=R^{12}\left(\theta^{\prime}-\theta\right) L_{n}^{2}\left(\theta^{\prime}\right) L_{n}^{1}(\theta)+O\left(\delta^{2}\right), \\
L_{n}(\theta)=\hat{L}(\theta, p(n), u(n)), \\
L^{1}=L \otimes 1, \quad L^{2}=1 \otimes L .
\end{gathered}
$$

Studying the known $L$-operators for the cases of $A_{1}^{(1)}[8]$ and $A_{2}^{(2)}[17]$, we observed that they could be written in the form $[26,30]$,

$$
\hat{L}(\theta, p, u)=e^{p}\left(1-i \delta m\left[\lambda e^{\mathrm{ad} u} I_{+}+\lambda e^{-\mathrm{ad} u} I_{-}\right]\right) e^{p},
$$

where $p=(p, \tilde{h}) ; u=(u, \tilde{h})$ and the rest of the notations were defined in (2.5), (3.27), (3.28). Note, that in the quasi-classical limit we have $\varphi \rightarrow 0, p \rightarrow-i \varphi \delta \pi(x)$ and

$$
\hat{L}(\theta, p, u) \simeq 1+\delta L_{c}(\theta, \pi, u)+O\left(\varphi^{2}\right),
$$

where $L_{c}(\theta, \pi, n)$ is the classical $L$-operator defined by relation (3.25).

It turns out that Eq. (5.5) works for all the algebras under consideration. We shall show below that the $L$-operator (5.5) and $R$-matrix (4.7) obey relation (5.4).

Let us enumerate some features of the $L$-operator (5.5),

$$
\hat{L}(\theta+\pi)=U^{-1} \hat{L}(\theta) U,
$$

where the explicit form of $U$ is presented in Tables 1 and 2,

$$
\hat{L}(\theta)(C(\varphi) B) \hat{L}^{t}(\theta-\varrho)(C(\varphi) B)^{-1}=1+O\left(\delta^{2}\right) .
$$

Here $t$ denotes taking the transpose of $L$ as a matrix in $\mathbb{C}^{N}$; the matrices $\mathrm{B}, C(\varphi)$ and the constant $\varrho$ are presented in Table 2 . For $\theta \rightarrow \pm i \infty$,

$$
\begin{gathered}
\left.\hat{L}(\theta)\right|_{\theta \rightarrow \pm i x}=J_{ \pm} e^{\mp 2 i \theta / g}+O(1), \\
J_{ \pm}=i m \delta e^{p} e^{ \pm \mathrm{ad} u} I_{ \pm} e^{p} .
\end{gathered}
$$

Equations (5.7) and (5.9) follow directly from the definition (5.5). Equation (5.8) is proved in Appendix B, using a new representation for $\varrho$ and the matrix $C(\varphi)$ in internal terms of the pair $\left(\mathscr{G}, A_{c}\right)$,

$$
C(\varphi)=\exp \left(i \varphi \sum_{a} c_{a} \tilde{h}_{a}\right)
$$

where $\tilde{h}_{a}, a=0, \ldots, r-1$, is a basis in $\mathscr{G}_{0}, c_{a}$ and $\varrho$ are determined by the equations

$$
-\left(\alpha_{j}, \alpha_{j}\right)+\left(\alpha_{j}, c\right)+\frac{2(\varrho+\Delta \varphi)}{g \varphi}=0 ; \quad j=0,1, \ldots, r,
$$


where $\alpha_{j}^{a}, \mathrm{j}=0, \ldots, r$, is the system of simple weights of ( $\mathscr{G}, A_{c}$ ) (see Sect. 2), $\Delta$ is defined in (3.15). The solution to system (5.12) always exists and is unique.

Now turn to the proof of Eq. (5.4). As in Sect. 3, the main idea will be to apply the Liouville theorem. Consider the difference $\Psi\left(\theta, \theta^{\prime}\right)$ between the right-hand side and left-hand side of Eq. (5.4) as a function of $\theta$ for fixed $\theta^{\prime}$. Since $L(\theta)$ is an entire function of $\theta$, then $\Psi\left(\theta, \theta^{\prime}\right)$ as well as $R\left(\theta-\theta^{\prime}\right)$ has simple poles at $\theta=-\theta^{\prime}+k \pi$; $\theta=-\theta^{\prime}-\varrho+k \pi ; k \in \mathbb{Z}$. As follows from (4.1) and (5.7), $\Psi\left(\theta, \theta^{\prime}\right)$ possesses automorphicity:

$$
\Psi\left(\theta+\pi, \theta^{\prime}\right)=(U \otimes 1)^{-1} \Psi\left(\theta, \theta^{\prime}\right)(U \otimes 1),
$$

and is therefore periodic in $\theta$ with the period $g \pi$. The residue of $R(\theta)$ at $\theta=0$ is of the form

$$
\left.\operatorname{Res} R(\theta)\right|_{\theta=0}=\sin 2 \varphi \cdot P,
$$

and at $\theta=-\varrho$ it is defined by (4.19). With the help of (5.8), (5.13), (5.14) one can show that the residues of $\Psi\left(\theta, \theta^{\prime}\right)$ are of the order of $O\left(\delta^{2}\right)$. Thus,

$$
\Psi\left(\theta, \theta^{\prime}\right)=\text { entire function of } \theta+O\left(\delta^{2}\right) .
$$

Consider next the limits $\theta \rightarrow \pm i \infty$. Using (4.15) and (5.9), we obtain

$$
\begin{gathered}
\left.\Psi\left(\theta, \theta^{\prime}\right)\right|_{\theta \rightarrow \pm i \infty}=\Psi_{1}^{ \pm}\left(\theta^{\prime}\right) e^{\mp 2 i \theta / g}+\Psi_{0}^{ \pm}\left(\theta^{\prime}\right)+O\left(e^{ \pm 2 i \theta / g}\right), \\
\Psi_{1}^{ \pm}\left(\theta^{\prime}\right)=\left[J_{ \pm} \otimes 1, R^{\mp}\left(1 \otimes e^{2 p}\right)\right]+O\left(\delta^{2}\right), \\
\Psi_{0}^{ \pm}\left(\theta^{\prime}\right)=\left(e^{2 p} \otimes \hat{L}\left(\theta^{\prime}\right)\right) R^{\mp}-R^{\mp}\left(1 \otimes \hat{L}\left(\theta^{\prime}\right)\right)\left(e^{2 p} \otimes 1\right)+\text { const } e^{\mp 2 i \theta^{\prime} / g}+O\left(\delta^{2}\right),
\end{gathered}
$$

where $J_{ \pm}$and $R^{ \pm}$are defined in (5.10) and (4.15), respectively. Using (2.9), (2.15), (5.1), one can easily exhibit that the commutator in (5.17) vanishes. Hence, up to the terms of the order of $O\left(\delta^{2}\right), \Psi\left(\theta, \theta^{\prime}\right)$ is independent of $\theta$ :

$$
\Psi\left(\theta, \theta^{\prime}\right)=\Psi_{0}^{+}\left(\theta^{\prime}\right)+O\left(\delta^{2}\right)=\Psi_{0}^{-}\left(\theta^{\prime}\right)+O\left(\delta^{2}\right) .
$$

In the same fashion it is proved that $\Psi\left(\theta, \theta^{\prime}\right)$ does not depend on $\theta^{\prime}$, i.e. that $\Psi^{+}\left(\theta^{\prime}\right)$ is a constant. The simplest way to calculate it is to let $\theta^{\prime} \rightarrow-i \infty$ in (5.18). Using (5.12) one obtains

$$
\Psi_{0}^{+}\left(\theta^{\prime}\right)=O\left(\delta^{2}\right) \text {. }
$$

It follows from (5.19), (5.20) that $\Psi\left(\theta, \theta^{\prime}\right) \equiv O\left(\delta^{2}\right)$, which does prove (5.4).

Note that in the quasi-classical limit (1.2), (5.6) relation (5.4) reduces to Eq. (3.30).

One more important observation is as follows. When proving (5.4), we used 1) automorphicity of the $R$-matrix $(4.7) ; 2)$ the expression for the residues (4.19), (5.14) and Eqs. (5.12), which define $C(\varphi)$ and $\varrho ; 3)$ the asymptotics (4.15). These properties define the $R$-matrix unambiguously. Thus, relations (5.4) and (5.5) may be taken as the basis to calculate the $R$-matrices $(4.7)^{6}$. This program was recently realized in $[30,39]$.

Using Eq. (5.4) it is not difficult to prove the unitarity of $R$-matrix (4.7). Applying Eq. (4.5) twice, we have

$$
\left[L^{1}(\theta) L^{2}\left(\theta^{\prime}\right), \hat{\Phi}\left(\theta-\theta^{\prime}\right)\right]=O\left(\delta^{2}\right)
$$

${ }^{6}$ This possibility was pointed out to the author by V. A. Fateev 
where $\hat{\Phi}(\theta)$ denotes left-hand side of Eq. (4.3). Expanding the product of two $L$-operators in a series in $\delta$, we obtain that $\hat{\Phi}(\theta)$ commutes with any matrix of the form $1 \otimes X$ and $X \otimes 1, X \in G$. Hence, $\hat{\Phi}(\vartheta)$ is proportional to the unity in $\mathbb{C}^{N} \otimes \mathbb{C}^{N}$.

\subsection{Integrable Models of Magnetics}

The $R$-matrices (4.7), (4.24) allow us to construct integrable models for magnetics in a standard way. The Hamiltonian is of the form

$$
\begin{gathered}
\mathbb{H}=\sum_{n} H_{n, n+1}, \\
H_{1,2}=i \partial /\left.\partial \theta\left(P R_{12}(\theta, \varphi)\right)\right|_{\theta=0} .
\end{gathered}
$$

When $\varphi$ is purely imaginary, $\mathbb{H}$ is real, and with account of (4.25), hermitian. The magnetics (5.21) are anisotropic generalizations of $s l(n), o(n), s p(n)$-invariant magnetics considered in [7].

\section{Factorized Representations for Quantum $R$-Matrices}

\subsection{Preliminary Remarks}

Let $f(z)$ be a meromorphic periodic function of $z, f(z+\pi)=f(z)$, bounded when $z \rightarrow \pm i \infty$, and having in the strip $0 \leqq \operatorname{Re} z<\pi$ simple poles at $z=a_{k}$, with the residues $c_{k}$ and zeros at $z=b_{k}, k=1, \ldots, r$. It is well known that such a function can be represented both as a sum of cotangents,

$$
f(z)=\sum_{k=1}^{r} c_{k} \operatorname{ctg}\left(z-a_{k}\right)+\text { const }
$$

and as a product of sines

$$
f(z)=\mathrm{const} \prod_{k=1}^{r} \frac{\sin \left(z-b_{k}\right)}{\sin \left(z-a_{k}\right)} .
$$

The trigonometric $R$-matrices, considered in Sect. 4 , are meromorphic quasiperiodic matrix functions of $\theta$. They are bounded at $\theta \rightarrow \pm i \infty$ and have simple poles only. The representations $(4.7),(4.24)$ for these $R$-matrices may be treated as a matrix analogue of the representation (6.1) for ordinary periodic functions. Note that the representation (4.7) allows one to check without difficulty the crossingsymmetry (4.2) of the corresponding $R$-matrices. However, checking the unitarity property (4.3) is not trivial.

In this section we shall construct new multiplicative representations for the $R$-matrices (4.7), (4.24), playing the same role as the representations (6.2) for ordinary functions. For that, we introduce elementary matrix multipliers (in general, noncommuting) which should be naturally considered as matrix generalizations of the functions $\sin z$ and $\cos z$. For these new representations, the unitarity of $R$-matrix becomes an easily checked feature.

\subsection{Matrix Generalizations of Trigonometric Functions}

As is known (see [43], Sect. 22.4), the function

$$
\operatorname{ctg} z=z^{-1}+\sum_{m=-\infty}^{\infty}\left[(z-m \pi)^{-1}+(m \pi)^{-1}\right]
$$


may be chosen as the basis for the theory of trigonometric functions. The prime at the sum sign means that the value $m=0$ is omitted. Then the function $\sin z$ is defined as the solution of the equation

$$
\frac{d}{d z} \ln f(z)=\operatorname{ctg} z
$$

with the initial condition,

$$
f(z)=z+O\left(z^{2}\right) ; \quad z \simeq 0 .
$$

Let us introduce the matrix analogues for $\operatorname{ctg}(z-\Delta)$ and $\sin (z-\Delta)$, where $\Delta$ is a constant. Let $\zeta(\theta)$ be a meromorphic matrix function in $\mathbb{C}^{N} \otimes \mathbb{C}^{N}$, with the following properties:

(i) Quasi-periodicity:

$$
\zeta(\theta+\pi)=(U \otimes 1) \zeta(\theta)(U \otimes 1)^{-1}=(1 \otimes U)^{-1} \zeta(\theta)(1 \otimes U),
$$

where $U$ is the matrix of finite order $g$, i.e. $U^{g}=1$.

(ii) $\zeta(\theta)$ has only simple poles when $\theta=k \pi+\Delta, k \in \mathbb{Z}$. For $\theta \simeq \Delta$

$$
\zeta(\theta)=\frac{M}{\theta-\Delta}+N+O(\theta-\Delta)
$$

where

$$
M^{2}=M
$$

$$
M N(1-M)=0 \text {. }
$$

(iii) $\zeta(\theta)$ is finite when $\theta \rightarrow \pm i \infty$,

$$
|\zeta(\theta)|<\infty, \quad \theta \rightarrow \pm i \infty .
$$

It is natural to consider the function $\zeta(\theta)$ as a matrix generalization of the function $\operatorname{ctg}(z-\Delta)$. The meaning of the requirements (6.8), (6.9) will clear up later on.

Define a matrix generalization of the sine function, $\sigma(\theta)$, as the solution of equation

$$
\frac{d \sigma}{d \theta}=\zeta(\theta) \sigma(\theta)
$$

with the initial condition

$$
\sigma\left(\theta_{0}\right)=\sigma_{0} ; \quad \theta_{0} \neq \Delta+k \pi, \quad k \in \mathbb{Z},
$$

$\sigma_{0}$ being a matrix in $\mathbb{C}^{N} \otimes \mathbb{C}^{N}$.

Let us show $\sigma(\theta)$ to be an entire function of $\theta$. It is sufficient to prove that $\sigma(\theta)$ is entire in the vicinity of singular points of Eq. (6.11), at $\theta=\Delta+k \pi, k \in \mathbb{Z}$. Let us consider Eq. (6.11) in the vicinity of the point $\theta=\Delta$.

Represent $\sigma(\theta)$ as

$$
\begin{gathered}
\sigma(\widetilde{\theta})=e^{M \ln \tilde{\theta}} \Sigma(\widetilde{\theta}), \\
e^{M \ln \tilde{\theta}}=1-M+M \tilde{\theta} ; \quad \tilde{\theta}=\theta-\Delta .
\end{gathered}
$$

The second equality has been obtained taking into account Eq. (6.8). The substitution of (6.7), (6.12) into (6.11) yields

$$
\frac{d \Sigma(\tilde{\theta})}{d \theta}=e^{-M \ln \tilde{\theta}}\left(\zeta(\theta) e^{M \ln \tilde{\theta}}-M\right) \Sigma(\widetilde{\theta})=\left(\frac{M N(1-M)}{\tilde{\theta}}+a_{0}+O(\tilde{\theta})\right) \Sigma(\widetilde{\theta}),
$$


Therefore $\Sigma(\theta)$ and, hence, $\sigma(\theta)$ are regular at $\tilde{\theta}=0$. Due to the quasi-periodicity of $\zeta(\theta)$, Eq. (6.6), the above reasoning hold for other singular points of Eq. (6.11), i.e. $\theta=\Delta+k \pi, k \in \mathbb{Z}$. The scheme of the above proving that $\sigma(\theta)$ is single-valued was borrowed from [32].

The function $\sigma(\theta)$ may be written as an ordered exponent,

$$
\begin{gathered}
\sigma(\theta)=\sigma\left(\theta, \theta_{0}\right) \sigma_{0}, \\
\sigma\left(\theta_{1}, \theta_{2}\right)=P \exp \left\{-\int_{\theta_{1}}^{\theta_{2}} \zeta(s) d s\right\}=\sigma\left(\theta_{1}\right) \sigma^{-1}\left(\theta_{2}\right) .
\end{gathered}
$$

The integration contour here is arbitrary because of the already proved uniqueness of $\sigma(\theta)$.

Note, it follows from the conditions (i)-(iii) on $\zeta(\theta)$ [without taking into account (6.9)] that

$$
\zeta(\theta)=Z(\theta-\Delta, M)+\delta .
$$

Here $\delta$ is a constant matrix in $\mathbb{C}^{N} \otimes \mathbb{C}^{N}$, commuting with $U \otimes 1$, whereas the function $Z(\theta, M)$ is defined by relation (3.10), with $P$ replaced by $M$,

$$
Z(\theta, M)=g^{-1} \sum_{k=0}^{g-1} \mathbf{U}^{k} M \mathbf{U}^{-k} \operatorname{ctg}((\theta-k \pi) / g) .
$$

\subsection{Two Types of $\zeta$ - and $\sigma$-Functions}

The above rather general definitions of the functions $\sigma(\theta)$ and $\zeta(\theta)$ were given without the connection with the structure of the $R$-matrices (4.7), (4.24). Now we are going to concretize these definitions.

Let $\widetilde{\mathscr{G}}=\left(\mathscr{G}, A_{c}\right)$ be one of the pairs from Tables 1 and 2 and $g, B, U, C(\varphi)$ take the corresponding values from Table 2. [We identify the matrix $U$ in (6.6) and (4.1).] Let us define two types of the functions $\zeta(\theta)$ with the same matrix $U$ but different residues (the function $\zeta_{2}$ is not defined for $A_{n-1}^{(1)}$ ):

$$
\begin{gathered}
\zeta_{1}(\theta)=Z\left(\theta, P_{-}\right)-i \delta_{1} / 2, \\
\zeta_{2}(\theta)=\left(Z(\theta-\Delta, K)-i \delta_{2}\right) / N .
\end{gathered}
$$

Here $Z(\theta, M)$ is defined in (6.18), $N$ is the matrix dimension of $\mathscr{G}, \Delta$ is given in (3.15), matrix $K$ is defined in (3.20),

$$
\begin{gathered}
P_{ \pm}=\frac{1}{2}(1 \pm P) ; \quad P_{ \pm}^{1}=P_{ \pm}, \\
\delta_{1}=i g^{-1} \sum_{k=1}^{g-1}\left(U^{-k} \otimes U^{k}\right) \operatorname{ctg}(k \pi / g), \\
\delta_{2}=i g^{-1} G_{0} \sum_{k=1}^{g-1}(U \otimes 1)^{-k} \operatorname{ctg}(k \pi / g) \operatorname{Tr}\left(U^{k}\right), \\
\tilde{\delta}_{1}=\delta_{1} G_{0} .
\end{gathered}
$$

The matrices $P$ and $P^{t}$ were defined by (1.6) and (3.21). The projection matrix in $\mathbb{C}^{N} \otimes \mathbb{C}^{N}, G_{0}$, entering into (6.24) is defined by

$$
\left(G_{\alpha}\right)_{i k}^{j l}=\delta_{i-\alpha, j} \delta_{k+\alpha . l} \delta_{j+l, N-1} ; \quad \alpha=0,1, \ldots, N-1 .
$$


Remember, the matrix indices run over the values $0,1, \ldots, N-1$, the indices $i, j$ refer to the first space $\mathbb{C}^{N}$, and the indices $k, l$ refer to the second one. Henceforth the indices in $\delta$-symbols are considered modulo $N$, i.e.

$$
\delta_{i j}=\delta_{i(\bmod N), j(\bmod N)} .
$$

The matrices $\delta_{1}, \delta_{2}$ may be calculated using the explicit form of $U$ from Table 1. Table 3 , given on page 498 , represents the vectors $\left\{d_{1,2}^{a}\right\}, a=0, \ldots, r-1$, related to $\delta_{1}, \delta_{2}$ as

$$
\tilde{\delta}_{1}=G_{0}\left(\Delta_{1} \otimes 1\right) ; \quad \delta_{2}=G_{0}\left(\Delta_{2} \otimes 1\right), \quad \Delta_{i}=\left(d_{i}, \tilde{h}\right),
$$

where $($,$) and h_{a}$ are defined by (4.12), (4.13).

Immediately from the definitions (6.19) and (6.20), it is easy to show that the functions $\zeta_{1}(\theta)$ possess classical unitarity

$$
\zeta_{1,2}(-\theta)=-P \zeta_{1,2}(\theta) P, \quad \delta_{1,2}=-P \delta_{1,2} P,
$$

and have the following asymptotic expansions at $\theta \rightarrow \pm i \infty$,

$$
\zeta_{1}(\theta)=\frac{i}{2}\left[\mp(1-\widetilde{P})-\delta_{1}\right]+O\left(e^{ \pm 2 i \theta / g}\right), \quad \zeta_{2}(\theta)=\frac{i}{N}\left[\mp \widetilde{K}-\delta_{2}\right]+O\left(e^{ \pm 2 i \theta / g}\right),
$$

where $\widetilde{P}$ and $\widetilde{K}$ are defined by (3.18) and (3.19).

Applying (6.19) - (6.23), one can easily verify that $\zeta_{1,2}(\theta)$ satisfies conditions (6.6)-(6.10). Hence, the functions $\sigma_{1,2}(\theta)$, defined via $\zeta_{1,2}(\theta)$ with the help of (6.11), are the entire functions of $\theta$.

Note, the function $\sigma_{1}(\theta)$ for the series $A_{n-1}^{(1)}$ (in a more general, elliptic case) was introduced in [32].

Using the definitions of the functions $\sigma_{1.2}(\theta)$ and their analytical properties, one can obtain their explicit expressions via the function (6.18). These calculations are performed in Appendix C.

Let us enumerate some properties of $\sigma_{1,2}(\theta)$ following from (6.6), (6.7), (6.11), (6.15), and (6.19)-(6.23):

$$
\sigma_{1,2}(\theta+\pi)=U \sigma_{1.2}(\theta) \Lambda_{1,2}
$$

where $\Lambda_{1.2}$ is independent of $\theta$,

$$
\begin{gathered}
\sigma_{1}(0)=P_{+} X_{1},\left.\quad \operatorname{Res} \sigma_{1}^{-1}(\theta)\right|_{\theta=0}=X_{1}^{\prime} P_{-}, \\
\sigma_{2}(\Delta)=(N-K) X_{2}, \quad \operatorname{Res} \sigma_{2}^{-1}(\theta)_{\theta=\Delta}=X_{2}^{\prime} K,
\end{gathered}
$$

where $X_{1}, X_{2}^{\prime}, X_{1}^{\prime}, X_{2}$ are some matrices.

Next, by virtue of (6.28), for the ratio of two functions $\sigma(\theta)$ defined in (6.16), one has

$$
\sigma_{1,2}\left(\theta_{1}, \theta_{2}\right)=P \sigma_{1,2}\left(-\theta_{1},-\theta_{2}\right) P \text {. }
$$

\subsection{Factorization of Quantum R-Matrices}

Here we present the factorized representations for the quantum $R$-matrices (4.7) and (4.24). The derivation of these representations is contained in Appendix D. The definition of the functions $\sigma_{1,2}$ are given in Sects. 6.2 and 6.3; the ratio of two functions $\sigma_{1,2}, \sigma_{1,2}\left(\theta_{1}, \theta_{2}\right)$, is defined in Eq. (6.16); the matrices $\delta_{1}, \delta_{2}$ are given in (6.22), (6.23). The matrix $C(\varphi)$ and the constant $\varrho$ entering into (4.7) are determined 
from (5.11) and (5.12) and also presented in Table $2 ; N$ is the matrix dimension of algebra $\mathscr{G}$.

Let us define the matrix

$$
\mathscr{C}=1-G_{0}+(C(\varphi) \otimes 1) G_{0},
$$

where $G_{0}$ is given by (6.25). As follows from (4.12), (4.13),

$$
P \mathscr{C} P=\mathscr{C}^{-1}
$$

Below it will be suitable to use the $R$-matrices $\tilde{R}(\theta)$ differing from (4.7) and (4.24) by the normalization

$$
\tilde{R}(\theta)=\sin \theta \quad R(\theta) / \sin (\theta+2 \varphi) .
$$

6.4.1. $\widetilde{\mathscr{G}}=A_{n-1}^{(1)} ; \quad \mathscr{G}=\operatorname{sl}(n)$.

$$
\begin{gathered}
\tilde{R}(\theta)=\mathscr{L} \sigma_{1}(\theta-2 \varphi, \theta+2 \varphi) \mathscr{L}, \\
\mathscr{L}=\exp \left(-i \varphi \delta_{1}\right) .
\end{gathered}
$$

This formula is a particular case of a more general result of [31] where an elliptic $R$-matrix [19] connected with algebra $\operatorname{sl}(N)$ was considered. Note that in the trigonometric limit the elliptic $R$-matrix of [31] goes not into $\widetilde{R}(\theta)$, but into the equivalent $R$-matrix $\mathscr{L}^{-1} \widetilde{R} \mathscr{L}^{-1}$. That is why Eq. (6.37) contains an additional factor $\mathscr{L}$ comparing with formula (4) from [31].

6.4.2. $\widetilde{G}=B_{n}^{(1)}, D_{n}^{(1)}, D_{n}^{(2)} ; \quad \mathscr{G}=o(2 n+1), o(2 n), o(2 n)$.

$$
\begin{gathered}
\widetilde{R}(\theta)=\mathscr{C}^{-1} \sigma_{2}(\theta-\varrho, \theta+2 \varphi) \mathscr{L} \sigma_{1}(\theta-2 \varphi, \theta+2 \varphi) \mathscr{L} \sigma_{2}(\theta-2 \varphi, \theta+\varrho) \mathscr{C}^{-1}, \\
\mathscr{L}=\exp \left(-i \varphi\left(\delta_{1}+\widetilde{\delta}_{1}\right)\right) ; \quad \varrho=(N-2) \varphi
\end{gathered}
$$

6.4.3. $\tilde{\mathscr{G}}=C_{n}^{(1)} ; \quad \mathscr{G}=s p(2 n)$.

$$
\begin{gathered}
\widetilde{R}(\theta)=\mathscr{C}^{-1} \sigma_{2}(\theta-\varrho, \theta-2 \varphi) \mathscr{L} \sigma_{1}(\theta-2 \varphi, \theta+2 \varphi) \mathscr{L} \sigma_{2}(\theta+2 \varphi, \theta+\varrho) \mathscr{C}^{-1}, \\
\mathscr{L}=\exp \left(i \varphi\left(\tilde{\delta}_{1}-\delta_{1}\right)\right) ; \quad \varrho=(N+2) \varphi .
\end{gathered}
$$

Note the difference of the signs in Eqs. (6.39), (6.40), and (6.41), (6.42).

6.4.4. $\widetilde{G}=A_{2 n}^{(2)} ; \quad \mathscr{G}=\operatorname{sl}(2 n+1)$.

$$
\begin{gathered}
\tilde{R}(\theta)=\mathscr{C}^{-1} \sigma_{2}(\theta-N \varphi) \mathscr{L} \sigma_{1}(\theta-2 \varphi, \theta+2 \varphi) \mathscr{L} \sigma_{2}^{-1}(\theta+N \varphi) \mathscr{C}^{-1}, \\
\mathscr{L}=\exp \left(-i \varphi \delta_{1}\right) \mathscr{C}^{-1} .
\end{gathered}
$$

The initial conditions for $\sigma_{2}(\theta)$ in (6.11) are chosen in the form

$$
\sigma_{2}(0)=\sigma_{0}=1-G_{0}+(-1)^{n}\left(G_{n}+G_{n+1}\right) / 2 .
$$

Here $G_{\alpha}$ are defined in (6.25). Equation (6.43) was reported in [25].

6.4.5. $\tilde{\mathscr{G}}=A_{2 n-1}^{(2)} ; \quad \mathscr{G}=\operatorname{sl}(2 n)$.

$$
\widetilde{R}(\theta)=\mathscr{C}^{-1} \sigma_{2}(\theta-N \varphi) \mathscr{L} F(\theta) \mathscr{L} \sigma_{2}^{-1}(\theta+N \varphi) \mathscr{C}^{-1} .
$$


The initial conditions for $\sigma_{2}(\theta)$ are chosen in the form

$$
\begin{gathered}
\sigma_{2}(0)=\sigma_{0}=1-G_{0}+\hat{\sigma}_{0}, \\
\left(\hat{\sigma}_{0}\right)_{i k}^{j l}=\delta_{i+k . N-1} \delta_{j+l, N-1} \Sigma_{k l},
\end{gathered}
$$

where the $2 n$ by $2 n$ matrix $\left\|\Sigma_{k l}\right\|$ is (the omitted matrix elements equal zero)

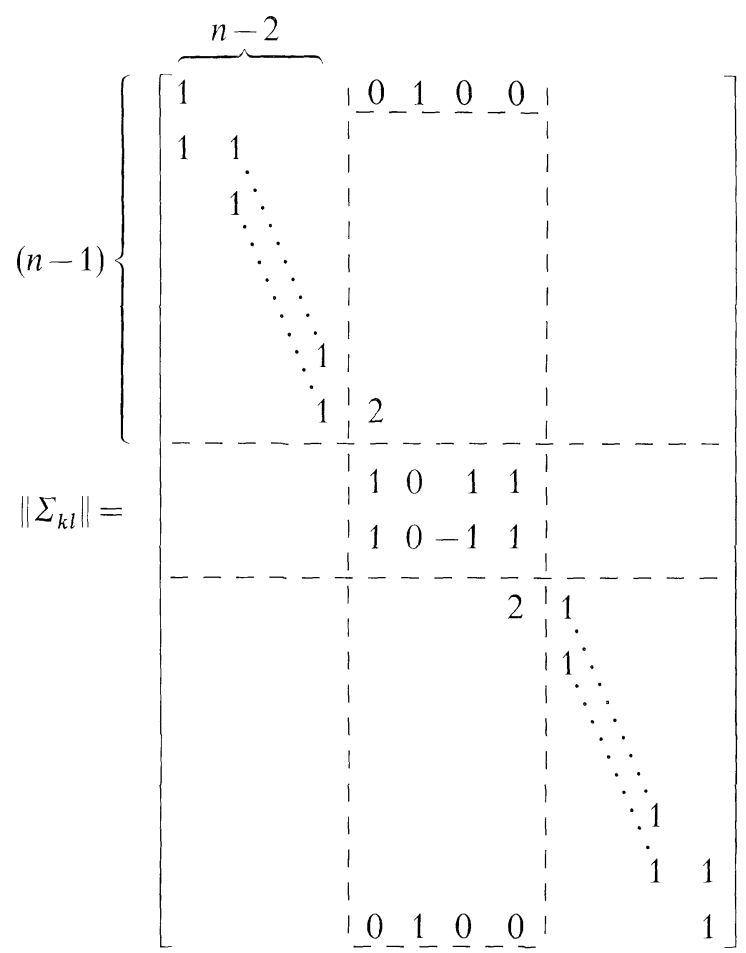

The matrix $\mathscr{L}$ is of the form

$$
\mathscr{L}=\mathscr{C} \exp \left[i \varphi\left(-\delta_{2}-\delta_{1}+\tilde{\delta}_{1}-\bar{\delta}_{1}\right)\right]
$$

where

$$
\begin{gathered}
\bar{\delta}_{1}=G_{0} i N^{-1} \sum_{k=1}^{N-1}\left(V^{-k} \otimes V^{k}\right) \operatorname{ctg} \frac{k \pi}{N}, \\
V=\operatorname{diag}\left(\omega^{n-1}, \omega^{n-2}, \ldots, \omega, 1,-1, \omega^{-1}, \ldots, \omega^{1-n}\right), \\
\omega=\exp (2 \pi i / N) .
\end{gathered}
$$

Next,

$$
F(\theta)=\left(1-G_{0}\right) \sigma_{1}(\theta-2 \varphi, \theta+2 \varphi)+G_{0} \bar{\sigma}_{1}(\theta-2 \varphi, \theta+2 \varphi),
$$

where $\bar{\sigma}_{1}\left(\theta_{1}, \theta_{2}\right)$ is defined by relations (6.11), (6.19) where $U, g, \delta_{1}$, and $P$ are replaced by $V, N, \bar{\delta}_{1}$, and $\bar{P}$ respectively, where

$$
\bar{P}=\sigma_{0}^{-1} P \sigma_{0}=1-G_{0}+G_{0} \hat{P} .
$$


In the same basis as Eq. (6.48), we have

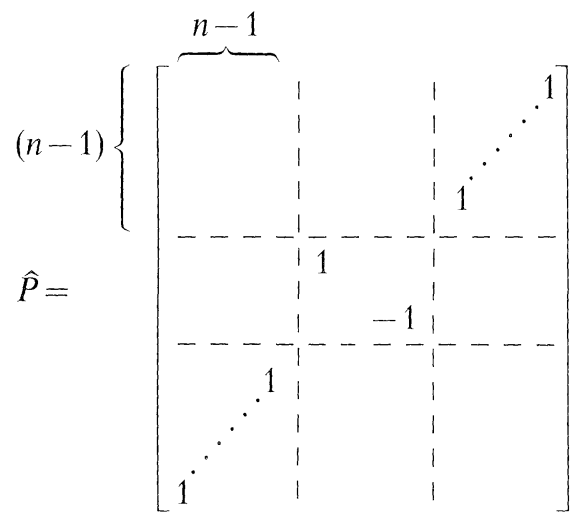

\section{Appendix A}

This appendix contains an explicit realization of relations (2.7)-(2.10). Remember that the discussed realizations of the algebras $s l(N), o(N), s p(N)$ are defined by (2.1), the Coxeter automorphisms are given in Table 1 . Let be the matrix dimension of $\mathscr{G}$, $\mathscr{G}_{0}$ be the subalgebra of $\mathscr{G}$, defined by $(2.6), r=\operatorname{dim} \mathscr{G}_{0}$. Define the $N$ by $N$ matrices

$$
\begin{gathered}
\tilde{h}_{a}=-e_{a, a}+e_{N-a-1, N-a-1}, \quad a=0, \ldots, r-1, \\
\left(e_{\alpha \beta}\right)_{i j}=\delta_{\alpha i} \delta_{\beta j},
\end{gathered}
$$

and the $r$-dimensional vectors

$$
\left(\varepsilon_{\alpha}\right)_{i}=\delta_{\alpha i} ; \quad i=1, \ldots, r .
$$

Equations (2.7)-(2.10) contain the elements $e_{i}, f_{i}$, and $\tilde{h}_{i}$ and the simple weights $\alpha_{i}$. We choose a normalization so that $f_{i}=e_{i}^{t}$. Below we list the sets $\left\{e_{i}, \alpha_{i}\right\}$.

1. Series $A_{2 n}^{(2)} ; \mathscr{G}=\operatorname{sl}(2 n+1), n \geqq 1, r=n$.

Generators:

$$
e_{0}=e_{2 n, 0}, \quad e_{i}=e_{i-1, i}+e_{2 n-i, 2 n+1-i}, \quad i=1, \ldots, n-1 ; \quad e_{n}=e_{n-1, n}+e_{n, n+1} .
$$

Simple weights:

$$
\alpha_{0}=2 \varepsilon_{1} ; \quad \alpha_{i}=-\varepsilon_{i}+\varepsilon_{i+1}, \quad i=1, \ldots, n-1 ; \quad \alpha_{n}=-\varepsilon_{n} .
$$

2. Series $A_{2 n-1}^{(2)}, \mathscr{G}=\operatorname{sl}(2 n), n \geqq 2, r=n$.

Generators:

$$
\begin{gathered}
e_{0}=e_{2 n-1,0}, \quad e_{i}=e_{i-1, i}+e_{2 n-1-i, 2 n-i}, \quad i=1, \ldots, n-1 ; \\
e_{n}=\left(e_{i-1, n}+e_{n-1, n+1}\right) .
\end{gathered}
$$

Simple weights:

$$
\alpha_{0}=2 \varepsilon_{1} ; \quad \alpha_{i}=-\varepsilon_{i}+\varepsilon_{i+1}, \quad i=1, \ldots, n-1, \quad \alpha_{n}=-\varepsilon_{n-1}-\varepsilon_{n} .
$$

3. Series $B_{n}^{(1)}, \mathscr{G}=o(2 n+1), n \geqq 1, r=n$. 
Generators:

$$
\begin{array}{cl}
e_{0}=\left(e_{2 n-1,0}-e_{2 n .1}\right), \quad e_{\imath}=e_{1-1, i}-e_{2 n-i, 2 n+1-i}, \quad i=1, \ldots, n-1 ; \\
e_{n}=e_{n-1, n}-e_{n, n+1} .
\end{array}
$$

Simple weights:

$$
\alpha_{0}=\varepsilon_{1}+\varepsilon_{2} ; \quad \alpha_{1}=-\varepsilon_{i}+\varepsilon_{i+1}, \quad i=1, \ldots, n-1 ; \quad \alpha_{n}=-\varepsilon_{n} .
$$

4. Series $C_{n}^{(1)}, \mathscr{G}=\operatorname{sp}(2 n), n \geqq 1, r=n$.

Generators:

$$
e_{0}=e_{2 n-1.0}, \quad e_{i}=e_{i-1, i}-e_{2 n-i-1.2 n-i}, \quad i=1, \ldots, n-1 ; \quad e_{n}=e_{n-1 . n} .
$$

Simple weights:

$$
\alpha_{0}=2 \varepsilon_{1}, \quad \alpha_{i}=-\varepsilon_{i}+\varepsilon_{i+1}, \quad \alpha_{n}=-2 \varepsilon_{n} .
$$

5. Series $D_{n}^{(1)}, \mathscr{G}=o(2 n), n \geqq 3, r=n$.

Generators:

$$
\begin{gathered}
e_{0}=\left(e_{2 n-2.0}-e_{2 n-1.1}\right), \quad e_{i}=e_{i-1, i}-e_{2 n-1-i, 2 n-i}, \quad i=1, \ldots, n-1 ; \\
e_{n}=\left(e_{n-2, n}-e_{n-1, n+1}\right) .
\end{gathered}
$$

Simple weights:

$$
\alpha_{0}=\varepsilon_{1}+\varepsilon_{2} ; \quad \alpha_{i}=-\varepsilon_{i}+\varepsilon_{i+1}, \quad \alpha_{n}=-\varepsilon_{n-1}-\varepsilon_{n} .
$$

6. Series $D_{n+1}^{(2)}, \mathscr{G}=o(2 n+2), n \geqq 2, r=n$.

Generators:

$$
\begin{gathered}
e_{0}=e_{n, 0}+e_{n+1,0}-e_{2 n+1, n}-e_{2 n+1, n+1}, \quad e_{i}=e_{i-1, i}-e_{2 n+1-1,2 n+2-i}, \\
i=1, \ldots, n-1 ; \quad e_{n}=e_{n-1, n}-e_{n-1, n+1}+e_{n, n+2}-e_{n+1, n+2} .
\end{gathered}
$$

Simple weights:

$$
\alpha=\varepsilon_{1}, \quad \alpha_{i}=-\varepsilon_{i}+\varepsilon_{i+1}, \quad \alpha_{n}=-\varepsilon_{n} .
$$

\section{Appendix B}

Here we shall obtain relation (5.8) for the quantum $L$-operator (5.5). List first some properties of the matrices $\tilde{h}_{a}, e_{k}, f_{k}$, entering into relations (2.7)-(2.10)

$$
B e_{k}^{t} B^{-1}=-\exp (+2 i \Delta / g) e_{k}, \quad B f_{k}^{t} B^{-1}=-\exp (-2 i \Delta / g) f_{k},
$$

where $B$ and $g$ are defined in Table 2, $A$ is defined in (3.15). For $G \neq \operatorname{sl}(N)$, when $\Delta=0$, the Eq. (C.1) are trivial consequences of the definitions (2.1). In the case of $G=\operatorname{sl}(N), \widetilde{G}=A_{2 n}^{(2)}, A_{2 n-1}^{(2)}$, when $\Delta=\pi / 2$, the Eqs. (C.1) follow from the definition (2.6) of the subspaces $G_{j}$ (remember that $e_{k} \in G_{1}, f_{k} \in G_{-1}$ ) and from the relation $\exp (2 i \Delta / g)=\omega=\exp (2 \pi i / h), h$ being the order for the Coxeter automorphism $A_{c}$. Next, for our realizations of $G$ and $A_{c}$ the matrices $\tilde{h}_{a} \in G_{0}$ are diagonal (see Appendix A). Therefore, Eq. (2.9) may be rewritten in the form

$$
\left(e_{k}\right)_{i j}\left(\tilde{h}_{i i}^{a}-\tilde{h}_{j j}^{a}\right)=\alpha_{k}^{a}\left(e_{k}\right)_{i j}, \quad\left(f_{k}\right)_{i j}\left(\tilde{h}_{i i}^{a}-\tilde{h}_{j j}^{a}\right)=-\alpha_{k}^{a}\left(f_{k}\right)_{i j}
$$


Besides, it follows from (A.1), (2.1), (5.3) that

$$
\tilde{h}_{a} B+B \tilde{h}_{a}=0 ; \quad e^{p} e^{(\alpha, u)} e^{-p}=e^{(\alpha, u-i \varphi \tilde{h})},
$$

where the notations are the same as in (3.22), (5.3), (5.5).

Let us come to the proof of (5.8). With the help of the definitions (3.28), (5.5) and the properties (B.1)-(B.3) one can easily show that

$$
B L^{t}\left(\theta-\varrho^{\prime}\right) B^{-1}=e^{-p}\left[1+\operatorname{im} \delta\left(e^{\operatorname{ad} u} \tilde{I}_{+} \lambda+e^{-\operatorname{ad} u} \widetilde{I}_{-} \lambda^{-1}\right)\right] e^{-p},
$$

where $Q^{\prime}$ is some constant, and the matrices $\widetilde{I}_{ \pm}$are defined by relations (3.28), with $e_{k}, f_{k}$, changed by $\tilde{e}_{k}, \widetilde{f}_{k}$,

$$
\begin{aligned}
& \tilde{e}_{k}=e_{k} \exp \left(-i \varphi\left(\alpha_{k}, \alpha_{k}\right)+2 i\left(\varrho^{\prime}+\Delta\right) / g\right), \\
& \tilde{f}_{k}=f_{k} \exp \left(+i \varphi\left(\alpha_{k}, \alpha_{k}\right)-2 i\left(\varrho^{\prime}+\Delta\right) / g\right) .
\end{aligned}
$$

The last two equalities may be written down as similarity transformations,

$$
\begin{gathered}
\tilde{e}_{k}=C^{\prime}(\varphi) e_{k} C^{\prime-1}(\varphi) ; \quad f_{k}=C^{\prime}(\varphi) f_{k} C^{\prime-1}(\varphi), \\
C^{\prime}(\varphi)=\exp (c, \tilde{h}),
\end{gathered}
$$

provided there exists an $r$-dimensional vector $c$ satisfying the system of equations

$$
-\left(\alpha_{k}, \alpha_{k}\right)+\left(c, \alpha_{k}\right)+2 i\left(\varrho^{\prime}+\Delta\right) / \varphi g=0, k=0, \ldots, r, \quad r=\operatorname{dim} \mathscr{G}_{0},
$$

where $\alpha_{K}$ are simple weights of $\widetilde{\mathscr{G}}$. Obviously, the vector $\frac{1}{2} c$ defines a point, equidistant with respect to $(r+1)$ points with the coordinates $\alpha_{k}, k=0, \ldots, r$, in an $r$-dimensional Euclidean space. Since $\alpha_{k} \neq \alpha_{l}$ when $K \neq l$, the vector $c$ and the constant $\varrho^{\prime}$ are determined by (B.8) unambiguously.

Using the explicit form of the system of simple weights $\left\{\alpha_{k}\right\}$, given in Appendix A, one can easily get convinced that the quantities $C^{\prime}(\varphi)$ and $\varrho^{\prime}$, defined via (B.7) and (B.8), coincide exactly with the result of [25] for $C(\varphi)$ and $\varrho$ given in Table 2,

$$
C^{\prime}(\varphi)=C(\varphi), \quad \varrho^{\prime}=\varrho .
$$

Taking into account (B.6), (B.9), we may rewrite (B.4) in the form

$$
C(\varphi) B L^{t}(\theta-\varrho) B^{-1} C^{-1}(\varphi)=e^{-p}\left[1+i m \delta\left(e^{\mathrm{ad} u} I_{+} \lambda+e^{-\mathrm{ad} u} I_{-} \lambda^{-1}\right)\right] e^{-p},
$$

whereof one can immediately obtain Eq. (5.8).

\section{Appendix C}

In this appendix we shall discuss the main properties of the functions $\sigma_{1}(\theta)$ and $\sigma_{2}(\theta)$, defined by the relations $(6.11),(6.19),(6.20)$.

1. The Function $\sigma_{1}(\theta)$. A particular choice of the initial condition $\left(6.11^{\prime}\right)$ is inessential for the representations (6.37), (6.39), (6.41), (6.43), since they contain only the ratio $\sigma_{1}\left(\theta_{1} ; \theta_{2}\right)$. We can use this freedom to simplify the calculations. It is convenient to replace $\left(6.11^{\prime}\right)$ by the initial condition of the form

$$
\sigma_{1}(\theta)=P_{+}+\theta P_{-}+O\left(\theta^{2}\right)
$$


where $P_{ \pm}$is defined in (6.21). This is possible because of the special structure of the expansion coefficients in (6.7) for $\zeta_{1}(\theta)$ (remember that $\Delta=0$ in this case). It follows from the definitions $(6.7),(6.22)$ that

$$
M=P_{-}, \quad N=-i P_{+} \delta_{1}=-i \delta_{1} P_{-},
$$

whereof it can be readily shown that in Eq. (6.14) $a_{0} \simeq P_{-} a_{0}^{\prime}$, and hence

$$
\Sigma(\theta)=\left(1+\theta P_{-} a_{0}^{\prime}\right) \Sigma(0)+O\left(\theta^{2}\right) .
$$

Substituting the last equation and Eq. (C.2) into (6.12), we get convinced that (C.1) is equivalent to the initial condition $\Sigma(0)=1$ for Eq. (6.14).

The function $\sigma_{1}(\theta)$, normalized by the condition (C.1), possesses both the general properties $(6.30),(6.31)$ with $M=P_{-}$, and the additional property [32],

$$
P \sigma_{1}(\theta)=\sigma_{1}(-\theta) \text {. }
$$

The latter is a consequence of (6.11) and the invariance of (C.1) under the transformations (C.3). Consider next the ratio of the form (6.16),

$$
\Phi_{1}(\theta)=\sigma_{1}(\theta-2 \varphi, \theta+2 \varphi)=\sigma_{1}(\theta-2 \varphi) \sigma_{1}^{-1}(\theta+2 \varphi) .
$$

The function $\Phi_{1}(\theta)$ is a meromorphic function of $\theta$ and possesses the following quasi-periodicity property

$$
\Phi_{1}(\theta+\pi)=(U \otimes 1) \Phi_{1}(\theta)(U \otimes 1)^{-1} .
$$

As follows from (6.31), it has poles at $\theta=-2 \varphi+k \pi, k \in \mathbb{Z}$, and

$$
\left.\operatorname{Res} \Phi_{1}(\theta)\right|_{\theta=-2 \varphi}=A_{1}(\varphi) P_{-} .
$$

Some analysis of the form of $\zeta_{1}$ shows that it is sufficient to choose the matrix $A_{1}(\varphi)$ to be diagonal. Then it follows from (C.5), (C.6), the definition (6.16) and the finiteness of $\zeta_{1}$ at $\theta \rightarrow \pm i \infty$, that

$$
\Phi_{1}(\theta)=A_{1}(\varphi) Z\left(\theta+2 \varphi, P_{-}\right)+B_{1}(\varphi) .
$$

Here $Z$ is defined in (6.18), and the matrices $A_{1}(\varphi)$ and $B_{1}(\varphi)$ are calculated by considering the limits of (C.7) when $\theta \rightarrow \pm i \infty$. Using (6.29), one obtains

$$
A_{1}(\varphi)=-2 e^{2 i \varphi \delta_{1}} \sin 2 \varphi \cos (2 \varphi \widetilde{P}), \quad B_{1}(\varphi)=e^{2 i \varphi \delta_{1}} \cos \left(2 \varphi\left(\widetilde{P}_{--} 1\right)\right),
$$

with $\delta_{1}$ defined by (6.22) and $\widetilde{P}$ defined by (3.18). In the derivation of (C.8) we exploited the property

$$
\widetilde{P}=\widetilde{P}^{3} .
$$

Similarly one may consider the function $\sin (\theta+2 \varphi) \Phi_{1}(\theta) / \sin (\theta-2 \varphi)$. Writing for it the representation of the type (C.7) one has

$$
\begin{aligned}
& \Phi_{1}(\theta)=\frac{\sin (\theta-2 \varphi)}{\sin (\theta+2 \varphi)}\left[-Z\left(\theta-2 \varphi, P_{+}\right) A_{1}(\varphi)+B^{\prime}(\varphi)\right], \\
& B^{\prime}(\varphi)=e^{2 i \varphi \delta_{1}} \cos [2 \varphi(\widetilde{P}+1)],
\end{aligned}
$$

where $A_{1}(\varphi)$ is given by (C.8). This representation, in particular, gives

$$
\Phi_{1}(2 \varphi)=-\sin ^{-1} 4 \varphi \quad P_{+} A_{1}(\varphi) .
$$


Let us obtain one more expression for $\Phi_{1}(\theta)$. It follows from (C.3) and (C.4) that

$$
\Phi_{1}(k \pi)=\mathbf{U}^{k} P \mathbf{U}^{-k}, \quad k \in \mathbb{Z} .
$$

Consider the function $\sin (\theta+2 \varphi) \Phi_{1}(\theta) / \sin \theta$. It is meromorphic and has poles at $\theta=k \pi, k \in \mathbb{Z}$, with the residues given by (C.12). At $\theta \rightarrow \pm i \infty$ its asymptotics is easily calculated by means of (C.7), (C.8). Therefore, it is easy to write for it an expression like (C.7). Thus, one comes to

$$
\Phi_{1}(\theta)=\frac{\sin \theta}{\sin (\theta+2 \varphi)}\left\{e^{2 i \delta_{1} \varphi}+(\cos 2 \varphi-1) \widetilde{P}^{2}+\sin 2 \varphi Z(\theta, P)\right\} .
$$

2. The Function $\sigma_{2}(\theta)$. The initial conditions become important for the function $\sigma_{2}(\theta)$, when one considers the series $A_{2 n}^{(2)}$ and $A_{2 n-1}^{(2)}$. They will be specified at the appropriate place (see Appendix D). Here we shall examine the following ratio of two functions $\sigma_{2}(\theta)$ for the case of $\mathscr{G}=o(N)$ and $\operatorname{sp}(N)$

$$
\Phi_{2}(\theta, \varphi)=\sigma_{2}(\theta-N \varphi, \theta)=\sigma_{2}(\theta-N \varphi) \sigma_{2}^{-1}(\theta),
$$

where $N$ is the matrix dimension of algebra $\mathscr{G}$. Repeating the analysis that has led to relations (C.7), (C.8), we obtain

$$
\Phi_{2}(\theta)=A_{2}(\varphi) Z(\theta, K)+B_{2}(\varphi),
$$

$K$ being defined in (3.20). The matrices $A_{2}(\varphi)$ and $B_{2}(\varphi)$ are of the form

$$
A_{2}(\varphi)=-\sin \varphi e^{i \varphi \delta_{2}} \cos (\varphi Y), \quad B_{2}(\varphi)=e^{i \varphi \delta_{2}} \cos \varphi \tilde{K},
$$

where $\tilde{K}, \delta_{2}$, and $G_{0}$ are defined by (3.19), (6.23), and (6.25)

$$
\tilde{K}=G_{0}+Y, \quad G_{0}^{2}=G_{0}, \quad G_{0} Y=Y, \quad Y^{3}=Y .
$$

It follows from (C.14) that

$$
\Phi_{2}(\theta, \varphi) \Phi_{2}(\theta-N \varphi,-\varphi)=1
$$

Putting here $\theta=N \varphi$ and substituting (C.15), we obtain

$$
Z(N \varphi, K) A_{2}(-\varphi) K=-A_{2}^{-1}(\varphi) B_{2}(\varphi) A_{2}(-\varphi) K .
$$

This relation will be used in Appendix D.

\section{Appendix D}

In this appendix we will prove Eqs. (6.37), (6.39), (6.44), (6.46).

1. $\widetilde{\mathscr{G}}=A_{n-1}^{(1)}, \mathscr{G}=\operatorname{sl}(n)$.

Equation (6.37) is a simple consequence of relations (4.24), (C.4), (C.13).

2. $\widetilde{G}=B_{n}^{(1)}, D_{n}^{(1)}, D_{n}^{(2)}, \mathscr{G}=o(2 n+1), o(2 n), o(2 n)$.

Let us calculate the function

$$
\hat{R}(\theta)=\sigma_{2}(\theta+2 \varphi, \theta-\varrho) \mathscr{C} R(\theta) \mathscr{C} \sigma_{2}(\theta+\varrho, \theta-2 \varphi),
$$

where $R(\theta)$ was given in (4.7), while all other notations were defined at the 
beginning of our Sect. 6. $\hat{R}(\theta)$ may have poles when $\theta= \pm \varrho+k \pi, \theta=2 \varphi+k \pi$, $\theta=k \pi$. Let us show that the residues of $\hat{R}(\theta)$ turn out to be nonzero only when $\theta=k \pi, k \in \mathbb{Z}$. It follows from (4.10) and (6.30) that

$$
\hat{R}(\theta+\pi)=\mathbf{U} \hat{R}(\theta) \mathbf{U}^{-1}
$$

Therefore, it is sufficient to consider only one pole of each series, e.g. $\pm \varrho, 2 \varphi, 0$. Assuming in (4.23) $\theta=-\varrho$ and applying (3.20) and (6.35), one obtains

$$
R(\varrho)=\mathscr{C}^{-1}(1-K / N) X,
$$

where $X$ is some matrix, $\mathscr{C}$ is defined by (6.34). Together with Eqs. (3.21) and (6.32), the last equality yields

$$
\left.\operatorname{Res} \hat{R}(\theta)\right|_{\theta=\varrho}=0 .
$$

By analogy, one can prove that

$$
\left.\operatorname{Res} \hat{R}(\theta)\right|_{\theta=-\varrho}=0 .
$$

For the consideration of the case $\theta=2 \varphi$ we shall employ the formula [which is valid for $G=o(N)]$

$$
\mathscr{C}=\exp \left(-i \varphi\left(\tilde{\delta}_{1}-\delta_{2}\right)\right)
$$

It is proved by an immediate substitution the values from Tables 2 and 3 , into (D.6). Using (3.11) and (C.15) we get

$$
\begin{gathered}
\left.\operatorname{Res} \hat{R}(\theta)\right|_{\theta=2 \varphi}=\sigma_{2}(4 \varphi, 4 \varphi-N \varphi) \mathscr{C} F, \\
F=R(2 \varphi) \mathscr{C} A_{2}(-\varphi) K,
\end{gathered}
$$

\begin{tabular}{|c|c|c|c|}
\hline \multirow{3}{*}{$\begin{array}{l}\tilde{\mathscr{G}} \\
A_{n-1}^{(1)} 1 \\
A_{2 n}^{(2)}\end{array}$} & & $\left\{d_{1}^{a}\right\},\left\{d_{2}^{a}\right\}$ & \\
\hline & \multirow{5}{*}{$\begin{array}{l}d_{1}^{(n-1)}=0 \\
d_{2}^{(n-1)}=0 \\
d_{1}^{0}=0 ; \\
d_{2}^{0}=0\end{array}$} & \multirow{7}{*}{$\begin{array}{l}d_{1}^{a}=(4 a+2-n) / n, \\
d_{1}^{a}=(4 a+1-2 n) /(2 n+1), \\
d_{2}^{a}=0, \\
d_{1}^{a}=(4 a+3-2 n) /(2 n-1), \\
d_{2}^{a}=(2 a+1) /(2 n-1), \\
d_{1}^{a}=(2 a-n) / n, \\
d_{2}^{a}=(a-n) / n, \\
d_{1}^{a}=(2 a-n+1) / n, \\
d_{2}^{a}=0, \\
d_{1}^{a}=(2 a+1-n) /(n-1), \\
d_{2}^{a}=(2 a+1-n) /(n-1), \\
d_{1}^{a}=(2 a+2-n) / n, \\
d_{2}^{a}=0,\end{array}$} & $0 \leqq a \leqq n / 2$ \\
\hline & & & $0 \leqq a \leqq n-1$ \\
\hline$A_{2 n-1}^{(2)}$ & & & $1 \leqq a \leqq n-2$ \\
\hline$B_{n}^{(1)}$ & & & $1 \leqq a \leqq n-1$ \\
\hline$C_{n}^{(1)}$ & & & $1 \leqq a \leqq n-1$ \\
\hline$D_{n}^{(1)}$ & $\begin{array}{l}d_{1}^{0}=d_{1}^{(n-1)}=0 \\
d_{2}^{0}=d_{2}^{(n-1)}=0 ;\end{array}$ & & $1 \leqq a \leqq n-1$ \\
\hline$D_{n}^{(2)}$ & $\begin{array}{l}d_{1}^{(n-1)}=0 \\
d_{2}^{(n-1)}=0\end{array}$ & & $0 \leqq a \leqq n-2$ \\
\hline
\end{tabular}

with $K$ and $A$ defined by (3.19) and (C.16), respectively. Substituting (4.7) into (D.8) and using (C.18), one has

$$
F=\left(R_{0}+\sin 2 \varphi Z(2 \varphi, P)\right) \mathscr{C} A_{2}(-\varphi) K+\sin 2 \varphi \mathscr{C} A_{2}^{-1}(\varphi) B_{2}(\varphi) A_{2}(-\varphi) K,
$$

Table 3. The values of $d_{1}$ and $d_{2}$ entering into Eqs. (6.27) 
where $R_{0}$ is given in (4.8). After some calculations involving the relations

$$
P_{+} K=K, \quad P_{-} K=0,
$$

where $P_{ \pm}$are defined in (6.21), we obtain that

$$
\begin{aligned}
F= & \exp \left(-i \tilde{\delta}_{1} \varphi\right)\left\{\left[\cos \left(2 \varphi t_{0}\right)+\cos (2 \varphi \tilde{P})\right] \sin \varphi \cos (\varphi Y)\right. \\
& \left.-\sin 2 \varphi \cos \left[\varphi\left(G_{0}+Y\right)\right]\right\} K .
\end{aligned}
$$

$G_{0}$ and $Y$ are specified in (6.25) and (C.17). Now it is not difficult to calculate the explicit form of the matrices $t_{0}, G_{0}, Y$ proceeding from the definitions (3.17), (3.18), (3.19), (C.17) and verify that for all the cases we are considering

$$
F \equiv 0 .
$$

Substituting the last equality into (C.7), one comes to

$$
\left.\operatorname{Res} \hat{R}(\theta)\right|_{\theta=2 \varphi}=0 \text {. }
$$

Thus, we have demonstrated that $\hat{R}(\theta)$ has no poles at $\theta \neq k \pi, k \in \mathbb{Z}$. The residues of $\hat{R}$ at $\theta=k \pi, k \in \mathbb{Z}$, are calculated with the help of (5.14), (6.35), (6.33),

$$
\left.\operatorname{Res} \hat{R}(\theta)\right|_{\theta=k \pi}=\sin 2 \varphi \mathbf{U}^{k} P \mathbf{U}^{-k},
$$

whereas the asymptotics for $\theta \rightarrow \pm i \infty$ is found applying (4.15), (6.29), (D.6). Restoring $\hat{R}(\theta)$ by (D.14), (D.15) with the help of the Liouville theorem and using (C.4), (C.13), we obtain that

$$
\sin \theta \hat{R}(\theta) / \sin (\theta+2 \varphi)=\mathscr{L} \sigma_{1}(\theta-2 \varphi, \theta+2 \varphi) \mathscr{L},
$$

where $\mathscr{L}$ was defined in (6.40). Equations (D.1) and (D.16) lead immediately to the representation (6.39).

3. $\widetilde{G}=C_{n}^{(1)}, \mathscr{G}=s p(2 n)$.

The proof of (6.41) is in a complete analogy with the above proof of Eq. (6.39). Note that in this case Eqs. (D.6) and (D.10) are replaced by the relations

$$
\begin{gathered}
\mathscr{C}=\exp \left(i \varphi \widetilde{\delta}_{1}\right), \\
P_{+} K=0, \quad P_{-} K=K .
\end{gathered}
$$

4. $\widetilde{G}=A_{2 n}^{(2)}, A_{2 n-1}^{(2)}, \mathscr{G}=\operatorname{sl}(2 n+1), \operatorname{sl}(2 n)$.

Remember, that the initial conditions for $\sigma_{2}(\theta)$ in Eq. (6.11) for the series $A_{2 n}^{(2)}$ and $A_{2 n-1}^{(2)}$ are given by the relations (6.45) and (6.47) respectively. Thus, we have

$$
\sigma_{2}(\theta)=\sigma_{2}(\theta, 0) \sigma_{0} \text {. }
$$

From (6.6) one has

$$
\begin{aligned}
& \sigma_{2}(\theta+\pi)=\mathbf{U} \sigma_{2}(\theta) \mathbf{V}^{-1}, \\
& \mathbf{U}=1-G_{0}+G_{0}(U \times 1), \\
& \mathbf{V}=1-G_{0}+G_{0}(V \times 1),
\end{aligned}
$$

where

$$
V^{-k}=\sigma_{0}^{-1} U^{-k} \sigma_{2}(k \pi, 0) \sigma_{0}
$$


Using (C.15), it can be shown

$$
\operatorname{Sp}\left(G_{0} V^{k}\right)=\left\{\begin{array}{ll}
0, & k \neq 0(\bmod N) \\
N, & k=0(\bmod N)
\end{array} .\right.
$$

It follows then that the eigenvalues of the matrix $\left(G_{0} V\right)$ are

$$
\lambda_{\alpha}=\exp (2 \pi i \alpha / N), \quad \alpha=0, \ldots, N-1 .
$$

For $\sigma_{0}$ in the form of (6.45) and (6.47) the matrix $V$ is diagonal and defined by the relation

$$
V=-U ; \quad \widetilde{\mathscr{G}}=A_{2 n}^{(2)},
$$

and by the relation (6.41) for $\widetilde{\mathscr{G}}=A_{2 n-1}^{(2)}$.

Turn now to the proof of (6.43). The matrices $(6.20),(6.21)$ in this case become

$$
K=\sum_{\alpha=0}^{N-1}(-\omega)^{-\alpha} G_{\alpha}, \quad \tilde{K}=G_{0},
$$

with the matrices $G_{\alpha}$ defined by (6.25). Substituting (D.27) into (6.20) and summing over $k$, one has

$$
\zeta_{2}(\theta)=N^{-1}\left\{G_{0} \operatorname{tg} \theta+i \cos ^{-1} \theta \sum_{\alpha=1}^{N-1}(-1)^{\alpha} G_{\alpha} \exp [i \theta(N-2 \alpha) / N]\right\} .
$$

This entails, in particular, that

$$
\zeta_{2}(0)=i N^{-1} \sum_{\alpha=1}^{N-1}(-1)^{\alpha} G_{\alpha} .
$$

The relation (C.15) in this case takes the form

$$
\sigma_{2}(\theta-N \varphi, \theta)=-N \sin \varphi \quad \zeta_{2}(\theta)+\cos \left(\varphi G_{0}\right) .
$$

Then it follows from (D.29), (D.30), (6.45) and the relation $G_{\alpha} G_{\beta}=G_{\alpha+\beta}$ that

$$
\sigma_{2}(\theta)=\frac{(-1)^{n}}{2}\left(e^{i \theta / N} G_{n}+e^{-i \theta / N} G_{n+1}\right)+1-G_{0} .
$$

Consider now the function

$$
\hat{R}(\theta)=\sigma_{2}^{-1}(\theta-N \varphi) \mathscr{C} R(\theta) \mathscr{C} \sigma_{2}(\theta+N \varphi) .
$$

By virtue of the properties of $\sigma_{2}(\theta), R(\theta)$ has no poles at $\theta=\pi / 2 \pm N \varphi+k \pi, k \in \mathbb{Z}$. The residues at poles at $\theta=k \pi$ are easy to calculate with the help of (5.14), (6.33), (D.20), (D.26),

$$
\left.\operatorname{Res} \hat{R}(\theta)\right|_{\theta=k \pi}=\sin 2 \varphi \mathbf{U}^{k} P \mathbf{U}^{-k} .
$$

Exploiting the asymptotic values following from (D.31)

$$
\left.\sigma_{2}(\theta)\right|_{\theta \rightarrow \pm i \infty}=\frac{(-1)^{n}}{2} G_{(N \pm 1) / 2} e^{\mp i \theta / N}+1-G_{0}+\ldots
$$


as well as Eqs. (3.17), (4.15) and

$$
G_{(N \pm 1) / 2} \mathscr{C} e^{ \pm 2 i \varphi \tilde{P}} \mathscr{C} G_{(N \mp 1) / 2}=\mathscr{C}^{-1} e^{ \pm 2 i \varphi \tilde{P}} \mathscr{C}^{-1} G_{0}
$$

we obtain

$$
\left.\hat{R}(\theta)\right|_{\theta \rightarrow \pm i \infty}=\mathscr{C}^{-1} e^{\mp 2 i \varphi \tilde{P}} \mathscr{C}^{-1},
$$

where $\widetilde{P}$ and $\mathscr{C}$ are defined by (3.18), (6.34). As follows from (D.33), (D.36), (C.13),

$$
\mathscr{C} \tilde{R}(\theta) \mathscr{C}=\sin (\theta+2 \varphi) \sin ^{-1} \theta e^{-i \varphi \delta_{1}} \sigma_{1}(\theta-2 \varphi, \theta+2 \varphi) e^{-i \delta \varphi_{1}} .
$$

Substituting (D.37) in (D.32), we obtain the representation (6.43). The proof of Eq. (6.46) is similar (though more cumbersome) and is therefore omitted here.

Acknowledgement. The author thanks A. G. Shadrikov for reading the manuscript and very useful comments.

\section{References}

1. Karowski, M., Thun, H., Truong, T., Weisz, P.: On the uniqueness of purely elastic $S$-matrix in $(1+1)$ dimension. Phys. Lett. B 67, 321-323 (1967);

Zamolodchikov, A.B.: Exact two-particle $S$-matrix of quantum solitons in sine-Gordon model. Pis'ma v ZhETF 25, 499-502 (1977)

2. Zamolodchikov, A.B., Zamolodchikov, A.B.: Factorized S-matrix in two dimension and exact solution of some relativistic field theory models. Ann. Phys. 120, 253-291 (1979)

3. Baxter, R.J.: Partition function of the eight-vertex lattice model. Ann. Phys. 70, 193-228 (1972)

4. Baxter, R.J.: Exactly solved models in statistical mechanics. London: Academic Press 1982

5. Yang, S.N.: $S$-matrix for the one-dimensional $N$-body problem with repulsive or attractive $\delta$-function interaction. Phys. Rev. 168, 1920-1923 (1968)

6. Belavin, A.A.: Exact solution of the two-dimensional model with asymptotic freedom. Phys. Lett. B 87, 117-121 (1979)

7. Reshetikhin, N.Yu.: $O(N)$ invariant quantum field theoretical models: Exact solution. Nucl. Phys. B 251 [FS13], 565-580 (1985)

8. Sklyanin, E.K., Takhtadjan, L.A., Faddeev, L.D.: Quantum inverse problem method I. Teor. Mat. Fiz. 40, 194-220 (1979)

9. Faddeev, L.D.: Quantum completely integrable models in field theory. Sov. Sci. Rev. C1, 107-155 (1980)

10. Drinfeld, V.G.: Hopf algebras and quantum triangle equations. Dokl. Akad. Nauk SSSR. 283, 1060-1064 (1985)

11. Sklyanin, E.K.: Quantum variant of the inverse scattering method. Zap. Nauchn. Semin. LOMI. 95, 55-128 (1980)

12. Perk, J.H., Schultz, C.L.: Diagonalization of the transfer matrix of a nonintersecting string model. Physica 122 A, 50-70 (1983)

13. Zamolodchikov, A.B., Zamolodchikov, Al.B.: Relativistic factorized $S$-matrix in twodimensions having $O(N)$ isotopic symmetry. Nucl. Phys. B 133, 525-535 (1978)

14. Berg, B., Karowski, M., Kurak, V., Weisz, P.: Factorized symmetric S-matrices in two dimensions. Nucl. Phys. B 134, 125-132 (1977)

15. Zamolodchikov, A.B., Fateev, V.A.: Model factorized $S$-matrix and an integrable Heisenberg chain with spin 1. Yad. Fiz. 32, 581-590 (1980) [English transl.: Sov. J. Nucl. Phys. 32, 298 (1980)]

16. Fateev, V.A.: Factorized $S$ matrix for particles with different parities and integrable 21 -vertex statistical model. Yad. Fiz. 33, 1419-1430 (1981) [English transl.: Sov. J. Nucl. Phys. 33, 761 (1980)]

17. Izergin, A.G., Korepin, V.E.: The inverse scattering method approach to the ShabatMikhailov model. Commun. Math. Phys. 79, 303-316 (1981) 
18. Cherednik, I.V.: On method of constructing factorized $S$ matrices in elementary functions. Teor. Mat. Fiz. 43, 117-119 (1980) [English transl.: Theor. Math. Phys. 43, 356 (1980)]

19. Belavin, A.A.: Discrete groups and the integrability of quantum systems. Funkts. Anal. Prilozh. 14, 18-26 (1980);

Belavin, A.A.: Dynamical symmetry of integrable quantum systems. Nucl. Phys. B 180 [FS2], 189-200 (1981)

20. Cherednik, I.V.: On properties of factorized $S$-matrices in elliptic functions. Yad. Fiz. 32, 549-557 (1982)

21. Bazhanov, V.V., Stroganov, Yu.G.: A new class of factorized $S$-matrices and triangle equations. Phys. Lett. B 105, 278-280 (1981)

22. Bazhanov, V.V., Stroganov, Yu.G.: Trigonometric and $S_{n}$ symmetric solution of triangle equations with variable on the faces. Nucl. Phys. B 205 [FS 5], 505-526 (1982)

23. Kulish, P.P., Sklyanin, E.K.: Solutions of the Yang-Baxter equations. Zap. Nauchn. Semin. LOMI. 95, 129-160 (1982) [English transl.: J. Soviet Math. 19, 1596 (1982)]

24. Belavin, A.A., Drinfeld, V.G.: Solutions of the classical Yang-Baxter equation for simple Lie algebras. Funkts. Anal. Prilozh. 16(3), 1-39 (1982) [English transl.: Funct. Anal. Appl. 16, 159 (1982)];

Belavin, A.A., Drinfeld, V.G.: Triangle equations and simple Lie algebras. Sov. Sci. Rev. 4C, 93-165 (1984)

25. Bazhanov, V.V.: Trigonometric solutions of the triangle equations and classical Lie algebras. Phys. Lett. 159 B, 321-324 (1985)

26. Bazhanov, V.V.: Exactly soluble models connected with simple Lic algebras. In: Special topics in gauge theories. Proc. XIX Int. Simp. Arenshoop, November, 1985, pp. 181-199

27. Bazhanov, V.V.: Quantum $R$-matrices and matrix generalizations of trigonometric functions. Preprint IHEP 86-39, Serpukhov: 1986 (in Russian) (submitted to Teor. Mat. Fiz.)

28. Leites, D.A., Serganova, V.V.: On solutions of the classical Yang-Baxter equations for simple Lie superalgebras. Teor. Mat. Fiz. 58, 26-37 (1984)

29. Bazhanov, V.V., Shadrikov, A.G.: Quantum triangle equations and Lie superalgebras. Preprint IHEP 86-43. Serpukhov: 1986 (submitted to Teor. Mat. Fiz.)

30. Jimbo, M.: Quantum $R$-matrix for the generalized Toda system. Commun. Math. Phys. 102, $537-547$ (1986)

31. Bazhanov, V.V., Stroganov, Yu.G.: On connection between the solutions of the quantum and classical triangle equations. In: Proc. 6th. Int. Sem. on high energy physics and field theory. Protvino 1, 51-53 (1983)

32. Takhtadjan, L.A.: Solutions of triangle equations with $Z_{n} \times Z_{n}$ symmetry and matrix analogs of Weierstrass $\zeta$ - and $\sigma$-functions. Zap. Nauchn. Semin. LOMI. 133, 258-276 (1984)

33. Reshetikhin, N.Yu., Faddeev, L.D.: Hamiltonian structures for integrable models of field theory. Teor. Mat. Fiz. 56, 311-321 (1983) [English transl.: Theor. Math. Phys. 56, 847 (1984)]

34. Kulish, P.P.: Quantum difference nonlinear Schrödinger equations. Lett. Math. Phys. 5, 191-197 (1981)

35. Olive, D.I., Turok, N.: Algebraic structure of Toda systems. Nucl. Phys. B 220 [FS 8], 491 (1983)

36. Leznov, A.N., Saveliev, M.V., Smirnov, V.G.: Group representation theory and integrable nonlinear systems. Teor. Mat. Fiz. 48, 3-12 (1981);

Bogoyavlensky, O.I.: On perturbations of the periodic Toda latice. Commun. Math. Phys. 51, 201-209 (1976)

37. Mikhailov, A.V., Olshanetsky, M.A., Perelomov, A.M.: Two-dimensional generalized Toda lattice. Commun. Math. Phys. 79, 473-488 (1981)

38. Jimbo, M., Miwa, T.: Classification of solutions to the star-triangle relations for a class of 3and 4-state IRF models. Nucl. Phys. B 257 [FS14], 1-18 (1985)

39. Jimbo, M.: Quantum $R$ matrix related to the generalized Toda system: an algebraic approach. Preprint RIMS-521, Kyoto: 1985

40. Babelon, O., de Vega, H.J., Viallet, C.M.: Solutions of the factorization equations from Toda field Theory. Nucl. Phys. B 190, 542 (1981) 
41. Izergin, A.G., Korepin, V.E.: Lattice version of quantum field theory models in two dimensions. Nucl. Phys. B 205 [FS 5], 401-413 (1982);

Izergin, A.G., Korepin, V.E.: The most general $L$-operator for the $R$-matrix of the XXX model. Lett. Math. Phys. 8, 259-265 (1984)

42. Babelon, O.: Representation of the Yang-Baxter algebra associated to Toda field theory. Nucl. Phys. B 230 [FS10], 241-249 (1983)

43. Whittaker, E.T., Watson, G.N.: A course of modern analysis. Cambridge: Cambridge University Press 1927

Communicated by Ya. G. Sinai

Received March 15, 1987 
